Org. Lett.

\title{
Synthesis and Structural Characterization of a Cyclen-Derived Molecular Cage
}

Alexander Ganß, ${ }^{\#}$ Raquel Belda, ${ }^{\ddagger}$ Javier Pitarch, ${ }^{\star}$ Richard Goddard,${ }^{\S}$ Enrique García-España, ${ }^{*}$ Stefan Kubik*,\#

\# Fachbereich Chemie - Organische Chemie, Technische Universität Kaiserslautern, ErwinSchrödinger-Straße, 67663 Kaiserslautern, Germany, Fax: +49-631-205-3921, Email: kubik@chemie.uni-kl.de

+ Instituto de Ciencia Molecular, Departamento de Química Inorgánica, Universidad de Valencia, C/ Catedrático José Beltrán 2, 46980, Paterna (Valencia) Spain $\S$ Max-Planck-Institut für Kohlenforschung, Kaiser-Wilhelm-Platz 1, 45470 Mülheim/Ruhr, Germany

\section{CONTENT}

Syntheses

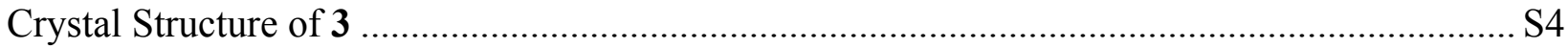

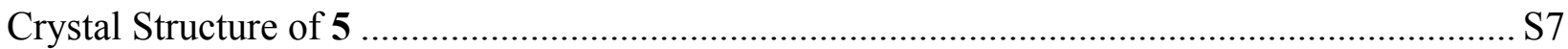

${ }^{1} \mathrm{H}$ NMR, ${ }^{13} \mathrm{C}$ NMR, and MALDI-MS Spectra of Products and Selected Intermediates............ S12

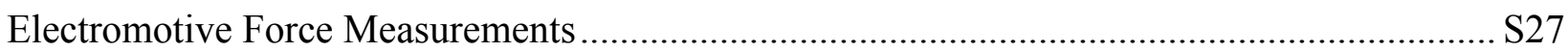

HR-ESI Mass Spectrometric Measurements ....................................................................... S30

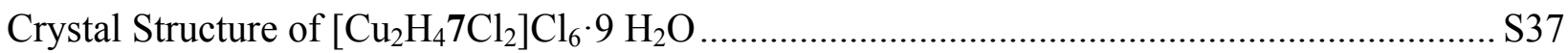

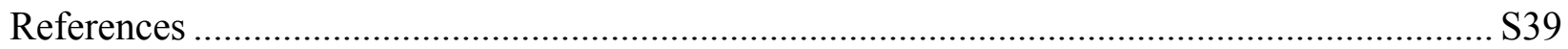


Syntheses

General details. Analyses were carried out as follows: melting points, Müller SPM-X 300; NMR, Bruker AVANCE III 400, Bruker AVANCE 600 digital NMR (peak assignments were confirmed by using H,H-COSY and HMQC spectra, spectra were referenced to the residual solvent signals $\left(\mathrm{CDCl}_{3}: \delta_{\mathrm{H}}=7.26 \mathrm{ppm}, \delta_{\mathrm{C}}=77.2 \mathrm{ppm}\right.$; DMSO- $\left.d_{6}: \delta_{\mathrm{H}}=2.50 \mathrm{ppm}, \delta_{\mathrm{C}}=39.5 \mathrm{ppm}\right)$; ESI-Ion Trap MS, Bruker Esquire 6000 and Bruker amaZon SL; elemental analysis, Elementar vario Micro cube. Chloroform, diethyl ether, and methanol used in the syntheses and purification steps were of p.a. grade $(>99.8 \%)$.

\section{1,4,7,10-Tetrakis(4-(1,3-dioxolan-2-yl)benzyl)-1,4,7,10-tetraazacyclododecane $2 .{ }^{1}$}

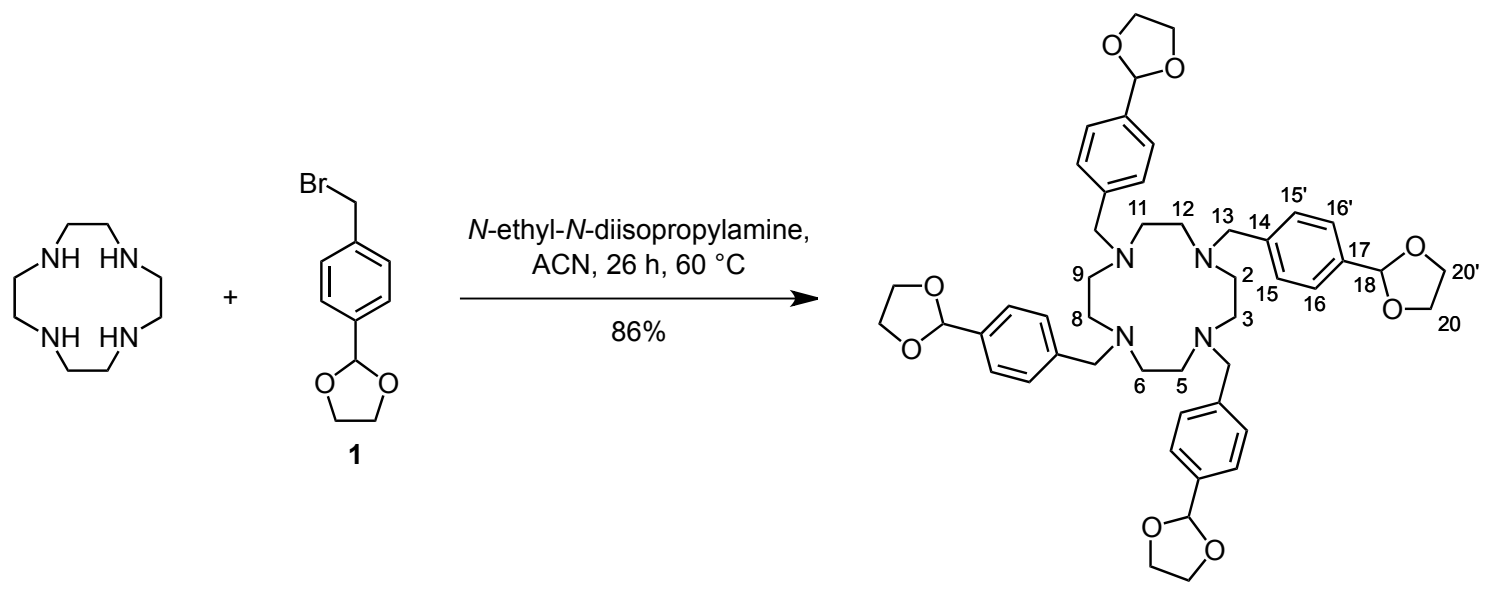

Cyclen $(0.25 \mathrm{~g}, 1.50 \mathrm{mmol})$ and $N$-ethyl- $N$-diisopropylamine $(1.88 \mathrm{~g}, 14.6 \mathrm{mmol})$ were dissolved in acetonitrile $(15 \mathrm{~mL})$ under an atmosphere of nitrogen. A solution of 2-[4-(bromomethyl)phenyl]1,3-dioxolane $\mathbf{1}^{2}(1.55 \mathrm{~g}, 6.38 \mathrm{mmol})$ in acetonitrile $(10 \mathrm{~mL})$ was added dropwise and the reaction mixture was stirred for $26 \mathrm{~h}$ at $60{ }^{\circ} \mathrm{C}$. The precipitated colorless solid was filtered off, washed with acetonitrile and dried in vacuo. Yield: $1.06 \mathrm{~g}$ (1.29 mmol, 86\%); mp.: 166-169 ${ }^{\circ} \mathrm{C} ;{ }^{1} \mathrm{H}$ NMR (600 $\mathrm{MHz}_{\mathrm{CDCl}}$ ) $\delta=7.37$ (s (apparent), 16H, H-15, H-15', H-16, H-16'), 5.80 (s, 4H, H-18), 4.00-4.14 (m $\left.\mathrm{m}_{\mathrm{AA}} \mathrm{BB}^{\prime}, 16 \mathrm{H}, \mathrm{H}-20, \mathrm{H}-20{ }^{\prime}\right), 3.42$ (s, 8H, H-13), 2.65 (s, 16H, cyclen protons) ppm; ${ }^{13} \mathrm{C}$ NMR (151 $\left.\mathrm{MHz}, \mathrm{CDCl}_{3}\right) \delta=141.4$ (C-14), 136.3 (C-17), 129.0 (C-15, C-15'), 126.4 (C-15, C-16'), 104.0 (C18), 65.4 (C-20, C-20'), 60.0 (C-13), 53.2 (cyclen carbon atoms) ppm; IR (KBr) v(bar) = $3053(\mathrm{w})$, 3029 (w), 2965 (m), 2943 (m), 2889 (s), 2808 (s), 2787 (s), 1622 (w), 1448 (m), 1370 (s), 1088 (s), $1070(\mathrm{~s}), 820(\mathrm{~m}), 790(\mathrm{~m}) \mathrm{cm}^{-1}$; MS (ESI) $\mathrm{m} / z(\%)=821.6[\mathrm{M}+\mathrm{H}]^{+}(100)$; CHN calculated for $\mathrm{C}_{48} \mathrm{H}_{60} \mathrm{~N}_{4} \mathrm{O}_{8}$ (M.W.: 821.03) C, 70.22; H, 7.37; N, 6.82; found: C, 70.47; H, 7.12; N, 6.97. 

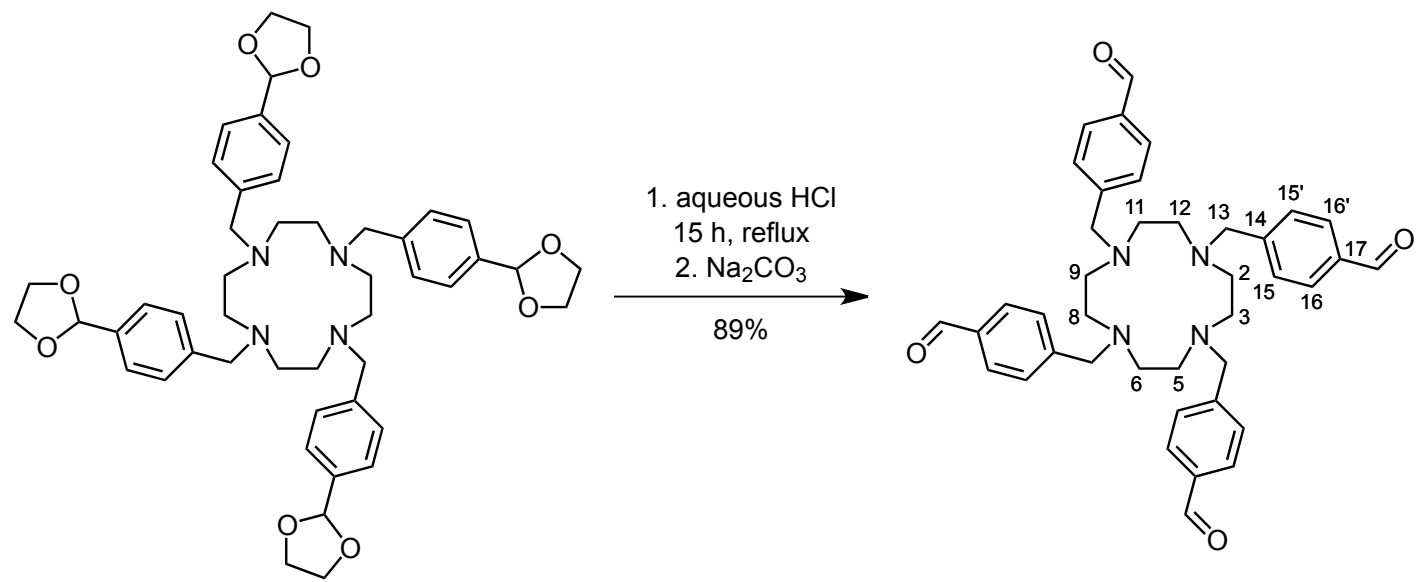

The product from the previous step $(1.08 \mathrm{~g}, 1.31 \mathrm{mmol})$ was dissolved in aqueous hydrochloric acid (10 mass- $\%, 80 \mathrm{~mL}$ ) and stirred at reflux for $15 \mathrm{~h}$. After cooling to $4{ }^{\circ} \mathrm{C}$ overnight, a crystalline solid formed that was filtered off. This solid was treated with $10 \%$ aqueous sodium carbonate solution $(30 \mathrm{~mL})$ and the resulting mixture was extracted three times with chloroform. Afterward, the combined organic layers were dried over $\mathrm{MgSO}_{4}$ and the solvent was evaporated. A suspension of the residue in methanol $(15 \mathrm{~mL})$ was refluxed for $1 \mathrm{~h}$. The colorless solid was filtered off, washed two times with methanol and dried in vacuo. Yield: $0.76 \mathrm{~g}(1.17 \mathrm{mmol}, 89 \%)$; $\mathrm{mp} .:$ 161-163 ${ }^{\circ} \mathrm{C} ;{ }^{1} \mathrm{H}$ NMR (400 MHz, $\left.\mathrm{CDCl}_{3}\right) \delta=9.96$ (s, 4H, CHO), 7.73-7.75 (mAA', 8H, H-16, H-16'), 7.517.53 (mXX', 8H, H-15, H-15'), 3.50 (s, 8H, H-13), 2.70 (s, 16H, cyclen protons) ppm; ${ }^{13} \mathrm{C}$ NMR (101 $\mathrm{MHz}_{\mathrm{CDCl}}$ ) $\delta=192.1$ (CHO), 147.5 (C-14), 135.5 (C-17), 129.8 (C-16, C-16'), 129.4 (C-15, C15'), 60.1 (C-13), 53.5 (cyclen carbon atoms) ppm; IR (KBr) v(bar) = 3367 (w), 3044 (w), 2965 (w), 2939 (w), 2884 (w), 2789 (s), 2737 (m), 1693 (s), 1605 (s), 1576 (m), 1447 (w), 1209 (s), 814 (m) $\mathrm{cm}^{-1}$; MS (ESI) $m / z(\%)=645.4[\mathrm{M}+\mathrm{H}]^{+}$; CHN calculated for $\mathrm{C}_{40} \mathrm{H}_{44} \mathrm{~N}_{4} \mathrm{O}_{4} \cdot 1 \mathrm{H}_{2} \mathrm{O}(\mathrm{M} . \mathrm{W} .: 662.83)$ C, 72.48; H, 7.00; N, 8.45; found: C, 72.47; H, 7.15; N, 8.52. 
Crystal structure of 3. Crystals of 3 were obtained by placing an open vial (4 mL) containing a solution of $\mathbf{3}$ in chloroform into a closed screw cap vial $(20 \mathrm{~mL})$ containing diethyl ether at $4{ }^{\circ} \mathrm{C}$.
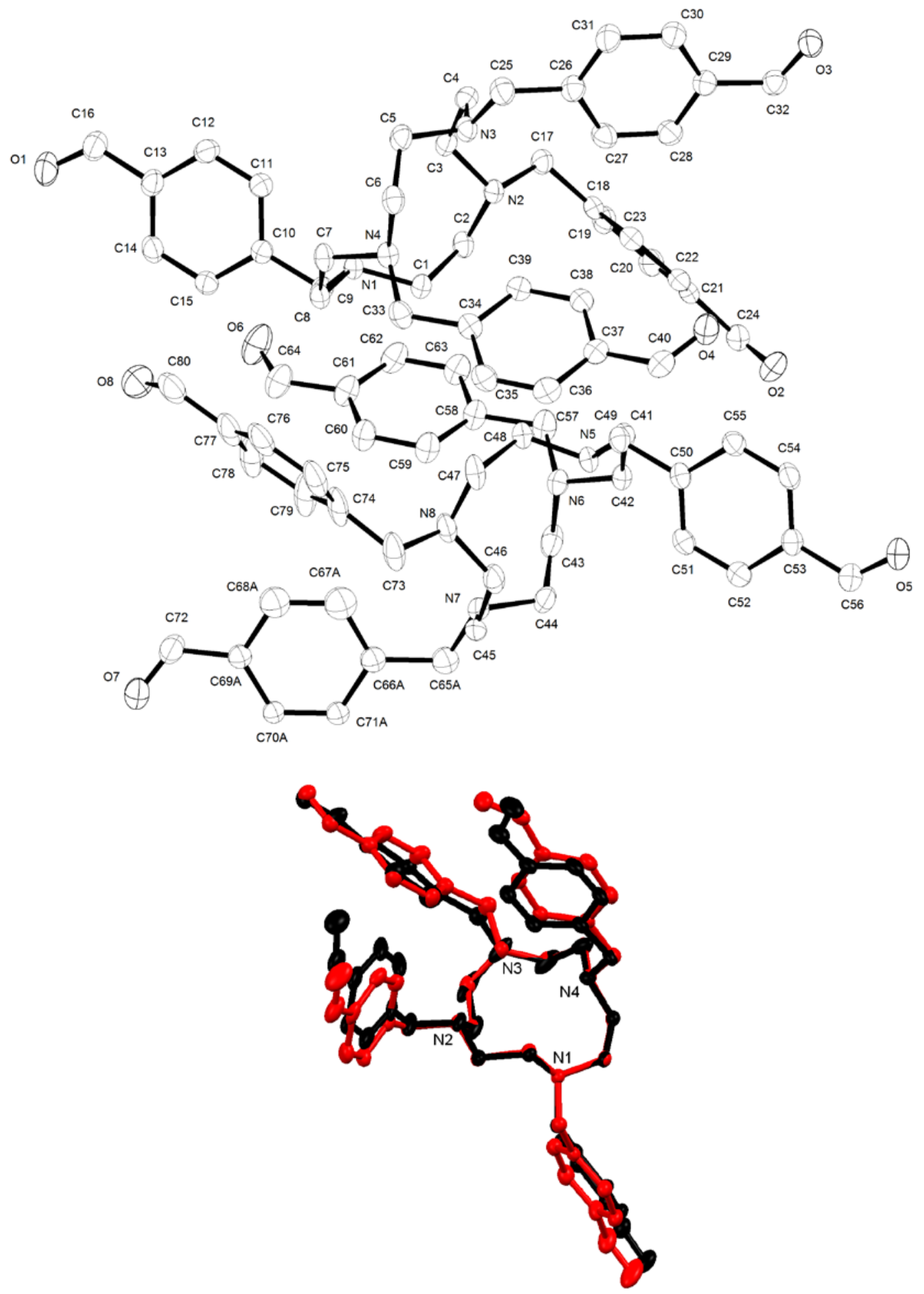

Figure S1: Two independent molecules of 3 in the unit cell. Superposition of the two independent molecules. Hydrogen atoms omitted for clarity. Ellipsoids are shown at the $50 \%$ probability level. 
Crystal data and structure refinement for 3. $\mathrm{C}_{40} \mathrm{H}_{44} \mathrm{~N}_{4} \mathrm{O}_{4}, M r=644.79 \mathrm{~g} \cdot \mathrm{mol}^{-1}$, colorless crystals, crystal size $0.18 \times 0.26 \times 0.36 \mathrm{~mm}^{3}$, triclinic, space group $P \overline{1}$, [No. 2], $a=11.5551(14) \AA$, $b=13.2390(17) \AA, c=24.552(3) \AA, \alpha=87.470(3)^{\circ}, \beta=82.055(3)^{\circ}, \gamma=66.377(3)^{\circ}, V=3407.9(7)$ $\AA^{3}, T=100 \mathrm{~K}, Z=4, D_{\text {calc }}=1.257 \mathrm{mg} \cdot \mathrm{m}^{-3}, \lambda=1.54178 \AA, \mu\left(\mathrm{Cu}-\mathrm{K}_{\alpha}\right)=0.649 \mathrm{~mm}^{-1}$, Gaussian absorption correction $\left(T_{\min }=0.83, T_{\max }=0.93\right), 1.87<\theta<67.137^{\circ}, 149939$ measured reflections, 11789 independent reflections $\left(R_{\text {int }}=0.0442\right), 10694$ reflections with $I>2 \sigma(I)$. Structure solved by direct methods (SHELXS) and refined by full-matrix least-squares (SHELXL) against $F^{2}$ to $R_{1}=$ $0.0466[I>2 \sigma(I)], w R_{2}=0.1229$ (all data), residual electron density $+0.57 /-0.43$ e $\AA^{-3}$. The crystal contains two independent molecules in the asymmetric unit. In one of the molecules a 4-formylbenzyl moiety is disordered over two positions (0.5:0.5). Disordered atoms were refined with isotropic atomic displacement parameters. CCDC-1431345.

Reactions between 3 and diamines (NMR scale). In a $4 \mathrm{~mL}$ vial 3 (12.0 $\mathrm{mg}, 18.1 \mu \mathrm{mol})$ was mixed with a solution of a diamine in $\mathrm{CDCl}_{3}(1.50 \mathrm{~mL}, 24 \mathrm{mM})$ by using a Hamilton syringe and trifluoroacetic acid (each $0.60 \mu \mathrm{L}$ ) was added as catalyst. ${ }^{1} \mathrm{H}$ NMR spectra of the mixtures were recorded after $1 \mathrm{~h}$ to check for the appearance of imine signals at ca. $8 \mathrm{ppm}$. The following diamines were used: 1,2-diaminoethane, 1,8-diaminooctane, triethylenetetramine, 2,2'-(ethylenedioxy)bis(ethylamine) (4), and benzene-1,2-diamine. In the case of the mixtures containing triethylenetetramine, 1,2-diaminoethane, or benzene-1,2-diamine precipitation of a solid was observed shortly after mixing. Clean product formation was observed in the presence of 4 . 


\section{Cyclen derivative 5 . $^{3}$}

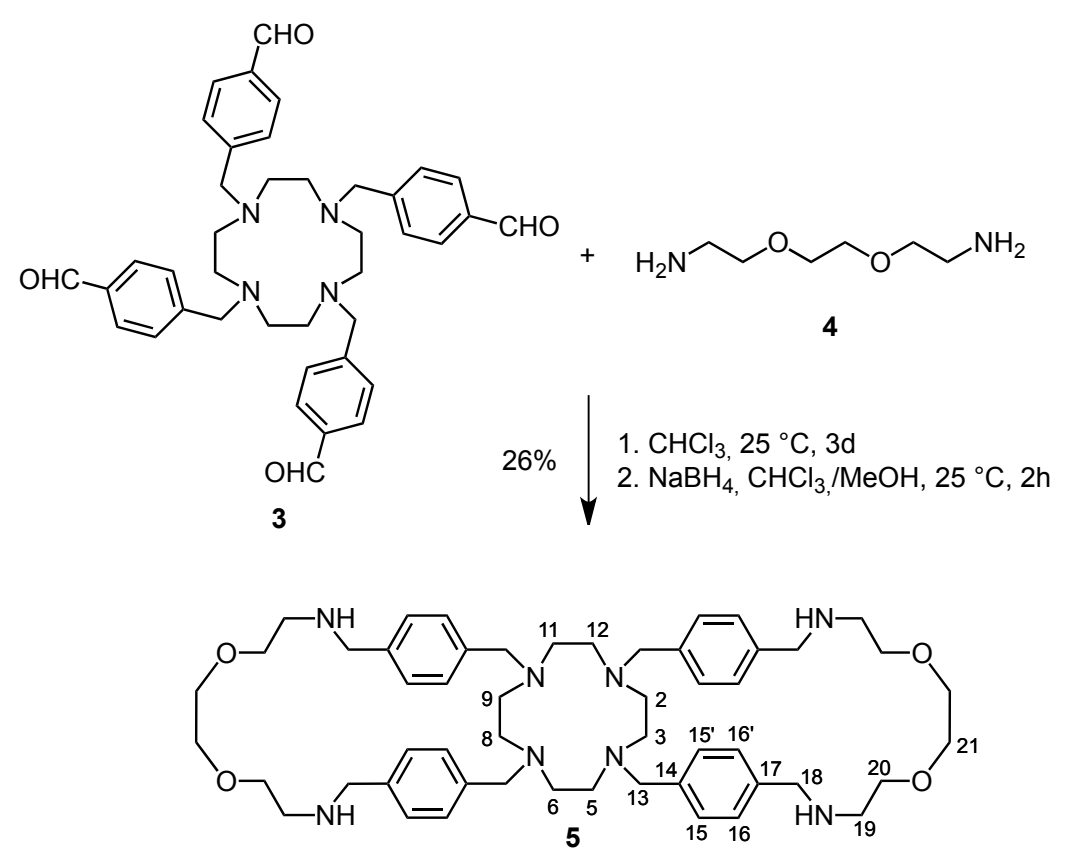

To a solution of $\mathbf{3}(0.49 \mathrm{~g}, 0.75 \mathrm{mM})$ in chloroform $(31 \mathrm{~mL})$ was added a solution of $4(0.23 \mathrm{~g}, 1.55$ $\mathrm{mmol})$ in chloroform $(31 \mathrm{~mL})$. The reaction mixture was stirred at room temperature for $3 \mathrm{~d}$. A solution of sodium borohydride $(1.14 \mathrm{~g}, 30.0 \mathrm{mmol})$ in methanol $(9 \mathrm{~mL})$ was added and stirring was continued for $2 \mathrm{~h}$ at room temperature. The reaction mixture was quenched by addition of water (50 $\mathrm{mL})$ and extracted three times with chloroform $(3 \times 80 \mathrm{~mL})$. The combined organic layers were washed with water, dried over $\mathrm{MgSO}_{4}$ and the solvent was evaporated. The residue was purified by semi-preparative HPLC (Supelco Analytical Ascentis ${ }^{\circledR} \mathrm{C} 18, \mathrm{CH}_{3} \mathrm{CN} / \mathrm{H}_{2} \mathrm{O}$-gradient: 0-5 min, 30\% $\mathrm{CH}_{3} \mathrm{CN}$; 5-35 min, linear gradient to $50 \% \mathrm{CH}_{3} \mathrm{CN}$; 35-45 min, 50\% $\mathrm{CH}_{3} \mathrm{CN}$; 45-50 min linear gradient to $90 \% \mathrm{CH}_{3} \mathrm{CN}$; $50-60 \mathrm{~min}, 90 \% \mathrm{CH}_{3} \mathrm{CN}$; $60-65$ min linear decrease to $30 \% \mathrm{CH}_{3} \mathrm{CN}$; 65 $85 \mathrm{~min} 30 \% \mathrm{CH}_{3} \mathrm{CN}$ ). Product fractions were collected between $30 \mathrm{~min}$ and $45 \mathrm{~min}$. After removal of the solvent the product was dissolved in methanol and filtered (syringe filter, pore size $0.20 \mu \mathrm{m}$ ). Removal of the solvent and drying in vacuo afforded the product as colorless solid. Yield: $171 \mathrm{mg}$ (195 $\mu$ mol, 26\%); m.p.: 153-156 ${ }^{\circ} \mathrm{C} ;{ }^{1} \mathrm{H}$ NMR (400 MHz, $\mathrm{CDCl}_{3}$ ) $\delta=7.22-7.32$ (underneath $\mathrm{CDCl}_{3}$ signal) (m $\left.\mathrm{m}_{\mathrm{AA}}, 8 \mathrm{H}, \mathrm{H}-15, \mathrm{H}-15^{\prime}\right)$, 7.07-7.17 (m $\left.\mathrm{mXX}^{\prime}, 8 \mathrm{H}, \mathrm{H}-16, \mathrm{H}-16^{\prime}\right), 3.78$ (s, 8H, H-18), 3.62 (br, 16H, H-20, H-21), 3.37 (s, 8H, H-13), 2.80 (m, 8H, H-19), 2.67 (s, 8H, cyclen protons), 2.63 (s, 8H, cyclen protons) ppm; ${ }^{13} \mathrm{C}$ NMR (101 MHz, CDCl3) $\delta=138.8$ (C-14), 138.6 (C-17), 129.1 (C-15, C15'), 127.8 (C-16, C-16'), $70.8+70.7$ (C-20, C-21), 60.0 (C-13), 53.9 (cyclen carbon atoms), 53.8 (C-18), 52.8 (cyclen carbon atoms), $48.9(\mathrm{C}-19)$; IR (KBr) v(bar) = $3422(\mathrm{~m}), 3329(\mathrm{~m}), 3048(\mathrm{w})$, 
$3011(w), 2944$ (s), 2873 (s), 2796 (s), $1511(w), 1450$ (s), 1139 (s), 1113 (s), 1078 (s), $820(\mathrm{~m}) \mathrm{cm}^{-}$ ${ }^{1}$; $\mathrm{MS}(\mathrm{ESI}) m / z(\%)=439.4[\mathrm{M}+2 \mathrm{H}]^{+}(20), 877.7[\mathrm{M}+\mathrm{H}]^{+}(100)$; $\mathrm{CHN}$ calculated for $\mathrm{C}_{52} \mathrm{H}_{76} \mathrm{~N}_{8} \mathrm{O}_{4} \cdot 2$ $\mathrm{H}_{2} \mathrm{O}$ (M.W.: 913.26) C, 68.39; H, 8.83; N, 12.27; found: C, 68.51; H, 8.60; N, 12.03.

Crystal structure of $\mathbf{5}$. Crystals of 5 were obtained by placing an open vial (4 mL) containing a solution of 5 in dichloromethane into a closed screw cap vial $(20 \mathrm{~mL})$ containing $n$-hexane at $4{ }^{\circ} \mathrm{C}$. Hydrogen atoms omitted for clarity. Ellipsoids are shown at the $50 \%$ probability level.

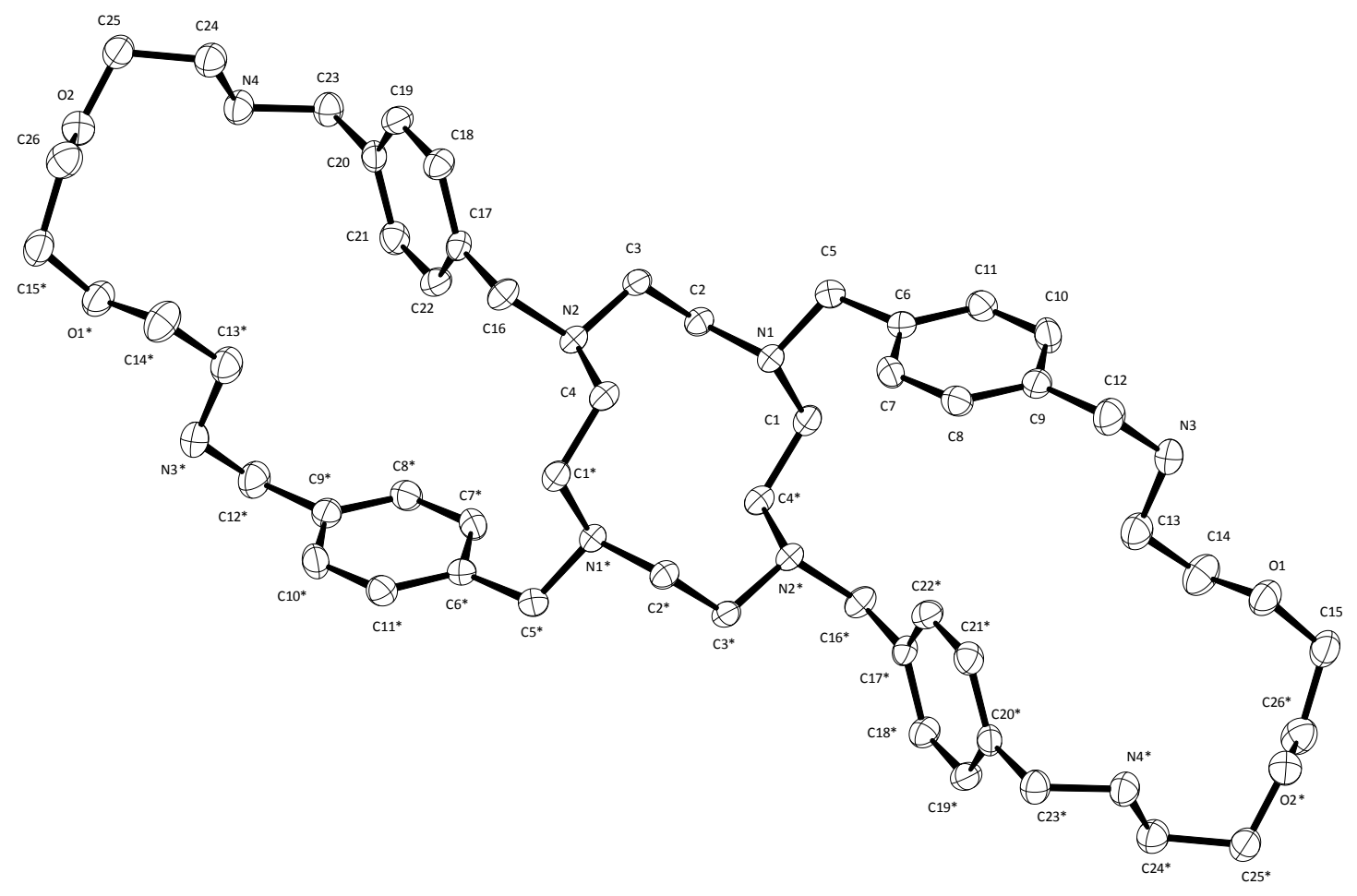

Figure S2: The crystal structure of 5. Hydrogen atoms omitted for clarity. Ellipsoids are shown at the $50 \%$ probability level.

Crystal data and structure refinement. $\mathrm{C}_{52} \mathrm{H}_{76} \mathrm{~N}_{8} \mathrm{O}_{4}, M_{\mathrm{r}}=877.20 \mathrm{~g} \cdot \mathrm{mol}^{-1}$, colorless crystals, crystal size $0.20 \times 0.17 \times 0.10 \mathrm{~mm}^{3}$, triclinic, space group $P \overline{1}$, [No. 2], $a=9.5396(4) \AA, b=$ 9.8037(4) $\AA, c=14.4253(6) \AA, \alpha=73.691(2)^{\circ}, \beta=71.815(1)^{\circ}, \gamma=74.944(2)^{\circ}, V=1208.03(9) \AA^{3}$, $T=100 \mathrm{~K}, Z=1, D_{\text {calc }}=1.206 \mathrm{mg} \cdot \mathrm{m}^{-3}, \lambda=1.54178 \AA, \mu\left(C u-K_{\alpha}\right)=0.606 \mathrm{~mm}^{-1}$, Gaussian absorption correction $\left(T_{\min }=0.91, T_{\max }=0.95\right), 3.30<\theta<66.85^{\circ}, 27404$ measured reflections, 4126 independent reflections $\left(R_{\text {int }}=0.0438\right), 3673$ reflections with $I>2 \sigma(I)$. Structure solved by direct methods (SHELXS) and refined by full-matrix least-squares (SHELXL) against $F^{2}$ to $R_{1}=$ $0.0377[I>2 \sigma(I)], w R_{2}=0.1029$ (all data), residual electron density $+0.23 /-0.19$ e $\AA^{-3}$. The short 
intermolecular $\mathrm{H} 3 \cdots \mathrm{H} 3$ distance of $2.03 \AA$ appears to be real, since $\mathrm{H} 3$ was located on a difference Fourier map and the refined N3-H3 distance and atomic displacement parameter for $\mathrm{H} 3$ are sensible. We cannot rule out that the center of symmetry is not obeyed by H3 and that the symmetry-related $\mathrm{H} 3$ attached to the neighboring N3 is directed elsewhere, although there was no evidence of this in the final difference Fourier synthesis. A similarly short H4 $\cdots \mathrm{H} 4$ distance of 2.35 $\AA$ is also observed for the $\mathrm{H}$ atom bonded to N4. CCDC-1431346.

\section{$3 \cdot \mathrm{ZnCl}_{2}$.}

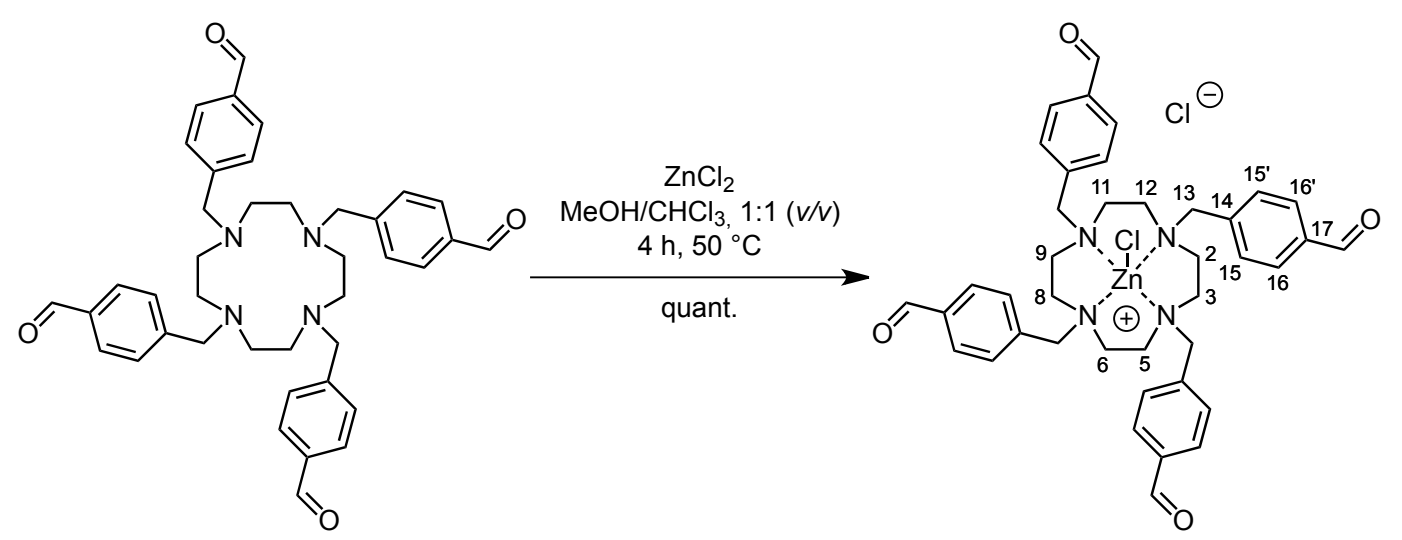

A solution of zinc chloride $(0.64 \mathrm{~g}, 4.72 \mathrm{mmol})$ in methanol $(60 \mathrm{~mL})$ was added to a stirred solution of $3(1.51 \mathrm{~g}, 2.34 \mathrm{mmol})$ in chloroform $(60 \mathrm{~mL})$ at room temperature. The resulting suspension was stirred for $4 \mathrm{~h}$ at $50{ }^{\circ} \mathrm{C}$. After cooling to room temperature the suspension was centrifuged and the solid was washed both with chloroform/methanol, 1:1 $(v / v)(30 \mathrm{~mL})$ and with methanol $(30 \mathrm{~mL})$. Subsequently, the solid was suspended in $n$-pentane $(60 \mathrm{~mL})$ and stirred for $17 \mathrm{~h}$ at room temperature. The colorless product was collected by filtration and dried in vacuo at $100{ }^{\circ} \mathrm{C}$. Yield: $1.83 \mathrm{~g}$ (2.34 mmol, 100\%); mp.: > $300{ }^{\circ} \mathrm{C} ;{ }^{1} \mathrm{H}$ NMR (400 MHz, DMSO-d $) \delta=10.10(\mathrm{~s}, 4 \mathrm{H}$, CHO), 8.02-8.05 (m $\mathrm{AA}^{\prime}, 8 \mathrm{H}, \mathrm{H}-16, \mathrm{H}-16$ '), 7.96-7.99 (m $\left.\mathrm{XX}^{\prime}, 8 \mathrm{H}, \mathrm{H}-15, \mathrm{H}-15 '\right)$, 4.50 (s, 8H, H-13), 3.05 (br, 8H, cyclen protons), 2.74 (br, 8H, cyclen protons) ppm; ${ }^{13} \mathrm{C}$ NMR (101 MHz, DMSO- $\left.d_{6}\right) \delta$ = $193.1(\mathrm{CHO}), 138.6$ (C-14), 136.0 (C-17), 132.6 (C-15, C-15'), 129.3 (C-16, C-16'), 55.5 (C-13), 47.5 (cyclen carbon atoms) ppm; IR (ATR) v(bar) = 3489 (w), 3057 (w), 2934 (w), 2881 (w), 1689 (s), 1607 (m), 1575 (w), 1475 (m), 1391 (w), 1309 (w), 1298 (w), 1214 (s), 1173 (s), 1069 (s), 1013 (w), 944 (m), 899 (m), 836 (s), 812 (s), 790 (s), 766 (m), 745 (s), 663 (m) cm-1; MS (ESI) m/z (\%) $=743.1 / 745.1[\mathrm{M}-\mathrm{Cl}]^{+}(100)$. 
General procedure for reactions between $\mathbf{3} \cdot \mathbf{Z n C l}_{\mathbf{2}}$ and diamines (NMR scale). An NMR tube containing a solution of $3 \cdot \mathrm{ZnCl}_{2}$ in DMSO- $d_{6}(200 \mu \mathrm{L}, c=36 \mathrm{mM})$ was diluted with DMSO- $d_{6}$ $(200 \mu \mathrm{L})$ and mixed with the solution of a diamine in DMSO- $d_{6}(200 \mu \mathrm{L}, 72 \mathrm{mM}) .{ }^{1} \mathrm{H}$ NMR spectra of the mixture were recorded after $1 \mathrm{~h}$ and $2 \mathrm{~d}$ to check for the appearance of imine signals at ca. 8 ppm. The following diamines were used: 1,2-diaminoethane, 1,8-diaminooctane, triethylenetetramine, 4, benzene-1,2-diamine, benzene-1,3-diamine, and benzene-1,4-diamine. Clean product formation was observed in the presence of $\mathbf{4}$ and 1,2-diaminoethane. The corresponding products were characterized by ${ }^{1} \mathrm{H}$ NMR spectroscopy and ESI-mass spectrometry.

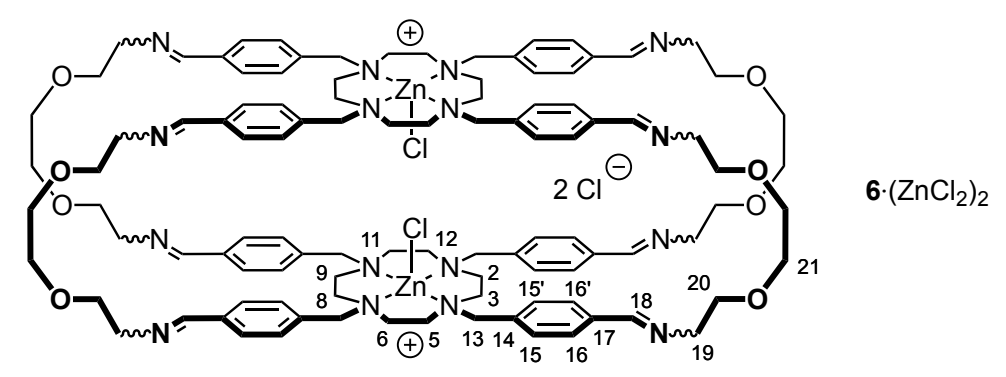

Bis(cyclen) $\left(\mathrm{ZnCl}_{2}\right)_{2}$ formed with $2,2^{\prime}-\left(\right.$ ethylenedioxy)bis(ethylamine) (imine form). ${ }^{1} \mathrm{H} \mathrm{NMR}$ $\left(400 \mathrm{MHz}, \mathrm{DMSO}-d_{6}\right) \delta=8.33$ (s, 8H, H-18), 7.61-7.71 (m $\left.\mathrm{mAA}^{\prime}, 16 \mathrm{H}, \mathrm{H}-15, \mathrm{H}-15^{\prime}\right), 7.46-7.58$ (mXX', 16H, H-16, H-16'), 4.26 (s, 16H, H-20), 3.49-3.62 (m, 64H, H-13, H-19, H-21), 2.50 (underneath DMSO signal) (br, 32H, H-2, H-3, H-5, H-6, H-8, H-9, H-11, H-12) ppm; MS (ESI) m/z (\%) = $969.4[\mathrm{M}-2 \mathrm{Cl}]^{2+}(100)$.

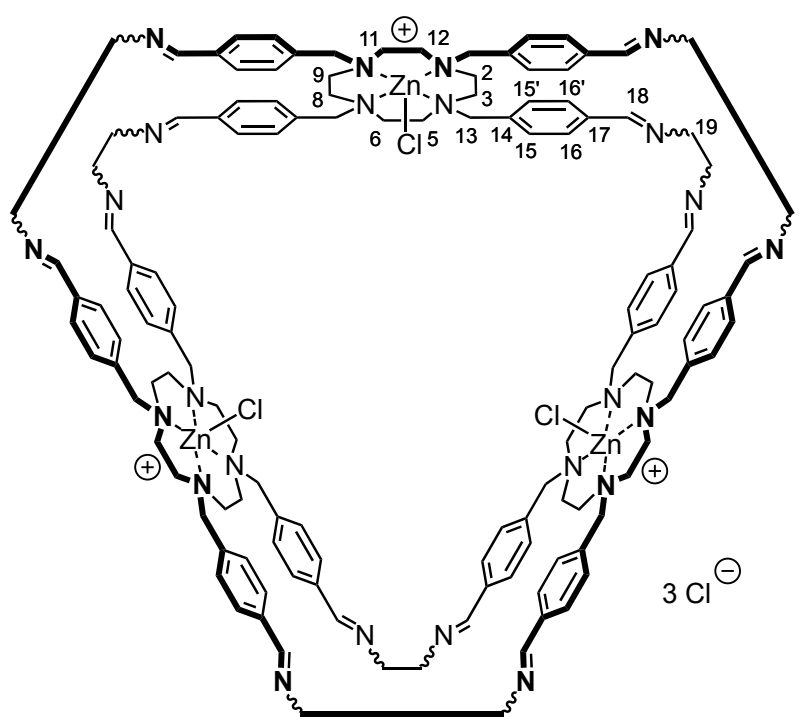

Tris(cyclen) $\left(\mathrm{ZnCl}_{2}\right)_{3}$ formed with 1,2-diaminoethane (imine form). ${ }^{1} \mathrm{H}$ NMR (400 MHz, DMSO- $\left.d_{6}\right) \delta=8.43$ (s, 12H, H-18), 7.70-7.75 (m $\left.\mathrm{AA}^{\prime}, 24 \mathrm{H}, \mathrm{H}-15, \mathrm{H}-15{ }^{\prime}\right), 7.63-7.69$ (mXX', 24H, H- 
16, H-16'), 4.26 (s, br, 24H, H-13), 4.02 (s, br, 24H, H-20), 2.94 (br, 12H, H-2, H-3, H-5, H-6, H-8, H-9, H-11, H-12), 2.60 (partly underneath DMSO signal) (br, 24H, H-2, H-3, H-5, H-6, H-8, H-9, H-11, H-12), 2.38 (partly underneath DMSO signal) (br, 12H, H-2, H-3, H-5, H-6, H-8, H-9, H-11, $\mathrm{H}-12) \mathrm{ppm}$; MS (ESI) $m / z(\%)=793.3[\mathrm{M}-3 \mathrm{Cl}]^{3+}(100)$.

\section{Bis(cyclen) 7.}
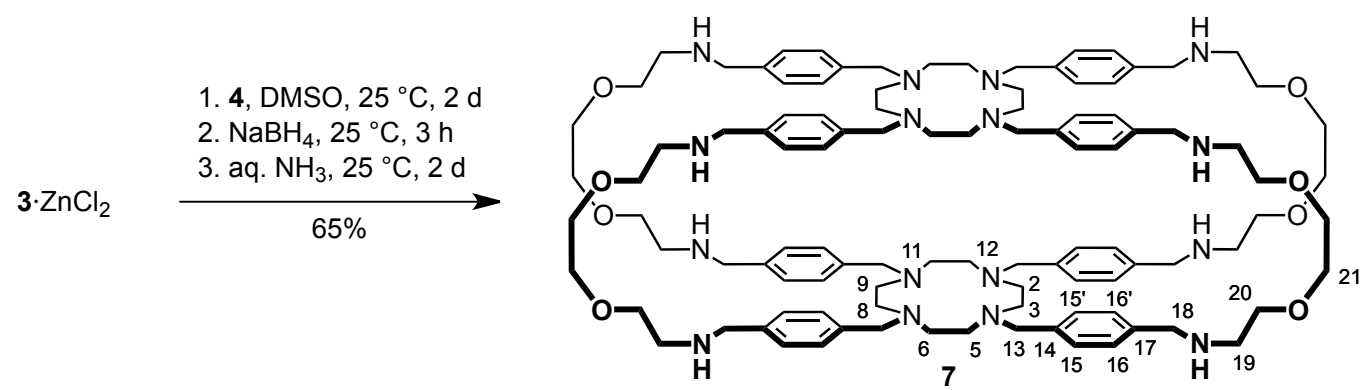

To a solution of $3 \cdot \mathrm{ZnCl}_{2}(1.61 \mathrm{~g}, 2.07 \mathrm{mmol})$ in DMSO (85 $\left.\mathrm{mL}\right)$ a solution of 2,2'(ethylenedioxy)bis(ethylamine) $4(0.61 \mathrm{~g}, 4.12 \mathrm{mmol})$ in DMSO $(85 \mathrm{~mL})$ was added and the reaction mixture was stirred at room temperature for $2 \mathrm{~d}$. Afterward, a solution of sodium borohydride $(3.10 \mathrm{~g}, 81.9 \mathrm{mmol})$ in methanol $(26 \mathrm{~mL})$ was added at room temperature under stirring and stirring was continued for $3 \mathrm{~h}$. The reaction was quenched by the addition of water (250 $\mathrm{mL})$ and the resulting mixture was extracted four times with chloroform $(4 \mathrm{x} 120 \mathrm{~mL})$. The combined organic layers were washed with water, dried over $\mathrm{MgSO}_{4}$ and the solvent was evaporated to afford a colorless solution. Residual DMSO was evaporated in vacuo. The residue was triturated with diethyl ether $(70 \mathrm{~mL})$ and the resulting suspension was stirred for additional $4 \mathrm{~d}$ at $25{ }^{\circ} \mathrm{C}$. The colorless solid obtained by filtration was dried in vacuo. Yield: $1.65 \mathrm{~g}$ of impure product.

The product was suspended in aqueous ammonia $(25 \%, 120 \mathrm{~mL})$ and the reaction mixture was stirred for $2 \mathrm{~d}$ at room temperature. It was extracted four times with chloroform and the combined organic layers were washed with water dried over $\mathrm{MgSO}_{4}$. Evaporation of the solvent afforded the crude product as a sticky residue. This crude product was triturated with diethyl ether $(50 \mathrm{~mL})$ followed by evaporation of the solvent. This procedure was repeated three times and the thus obtained solid was once again suspended in diethyl ether $(50 \mathrm{~mL})$. This suspension was stirred at room temperature for $16 \mathrm{~h}$. Filtration and drying in vacuo at $50{ }^{\circ} \mathrm{C}$ afforded the product as a colorless solid. Yield: $1.18 \mathrm{~g}(674 \mu \mathrm{mol}, 65 \%)$; mp.: 131-134 ${ }^{\circ} \mathrm{C} ;{ }^{1} \mathrm{H}$ NMR $\left(400 \mathrm{MHz}, \mathrm{CDCl}_{3}\right) \delta=$ 
7.19-7.23 (m $\left.\mathrm{mAA}^{\prime}, 16 \mathrm{H}, \mathrm{H}-15, \mathrm{H}-15^{\prime}\right)$, 7.12-7.16 (mXX', 16H, H-16, H-16'), 3.70 (s, 16H, H-18), 3.57 (s, 32H, H-20, H-21), 3.33 (s, 16H, H-13), 2.73-2.75 (m, 16H, H-19), 2.62 (s, 32H, cyclen protons) ppm; ${ }^{13} \mathrm{C}$ NMR (101 MHz, $\mathrm{CDCl}_{3}$ ) 138.7, 138.6 (C-14, C-17), 129.2 (C-15), 128.0 (C-16), 70.7, 70.4 (C-20, C-21), 59.8 (C-13), 53.9 (C-18), 52.4 (cyclen carbon atoms), 48.8 (C-19) ppm; IR $($ ATR $) v($ bar $)=3322(\mathrm{w}), 3013(\mathrm{w}), 2867(\mathrm{~s}), 2796(\mathrm{~s}), 1672(\mathrm{w}), 1511(\mathrm{~m}), 1449(\mathrm{~s}), 1418(\mathrm{~m})$, 1353 (s), 1282 (m), 1104 (s), 1078 (s), 1051 (m), 816 (m), 790 (m), 753 (m) cm ${ }^{-1}$; MS (ESI) m/z $(\%)=878.0[\mathrm{M}+2 \mathrm{H}]^{2+}(100), 931.4[\mathrm{M}+2 \mathrm{HCl}+2 \mathrm{H}]^{2+}(6), 1755.0[\mathrm{M}+\mathrm{H}]^{+}(4) ; \mathrm{CHN}$ calculated for $\mathrm{C}_{104} \mathrm{H}_{152} \mathrm{~N}_{16} \mathrm{O}_{8} \cdot 1 \mathrm{H}_{2} \mathrm{O}$ (M.W.: 1772.44) C, 70.47; H, 8.76; N, 12.64; found: C, 70.28; H, 8.76; N, 12.71 . 
${ }^{1} \mathrm{H}$ NMR: $2\left(600 \mathrm{MHz}, \mathrm{CDCl}_{3}, 25^{\circ} \mathrm{C}\right)$.

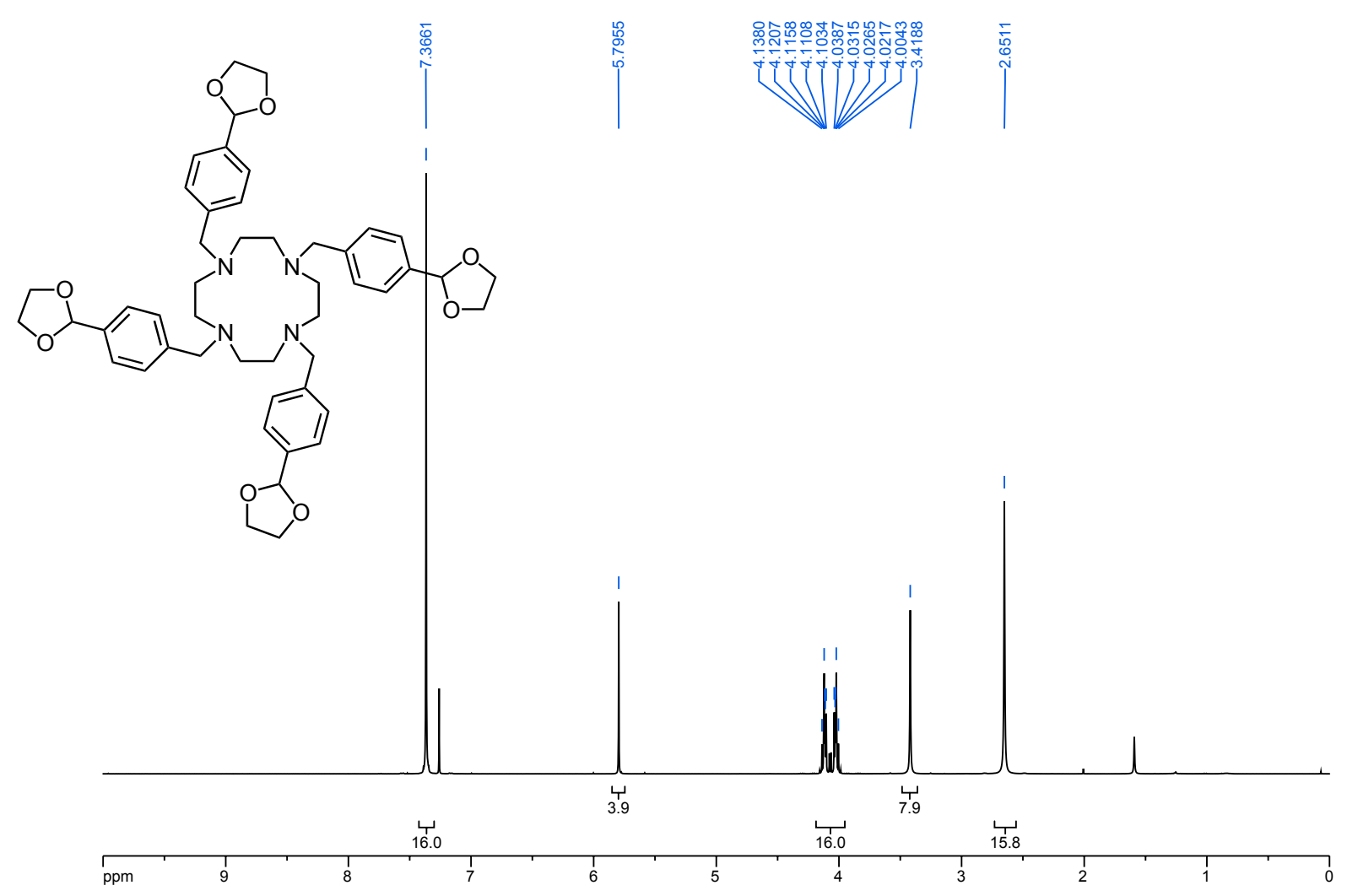

${ }^{13} \mathrm{C}$ NMR: 2 (151 MHz, $\left.\mathrm{CDCl}_{3}, 25^{\circ} \mathrm{C}\right)$.

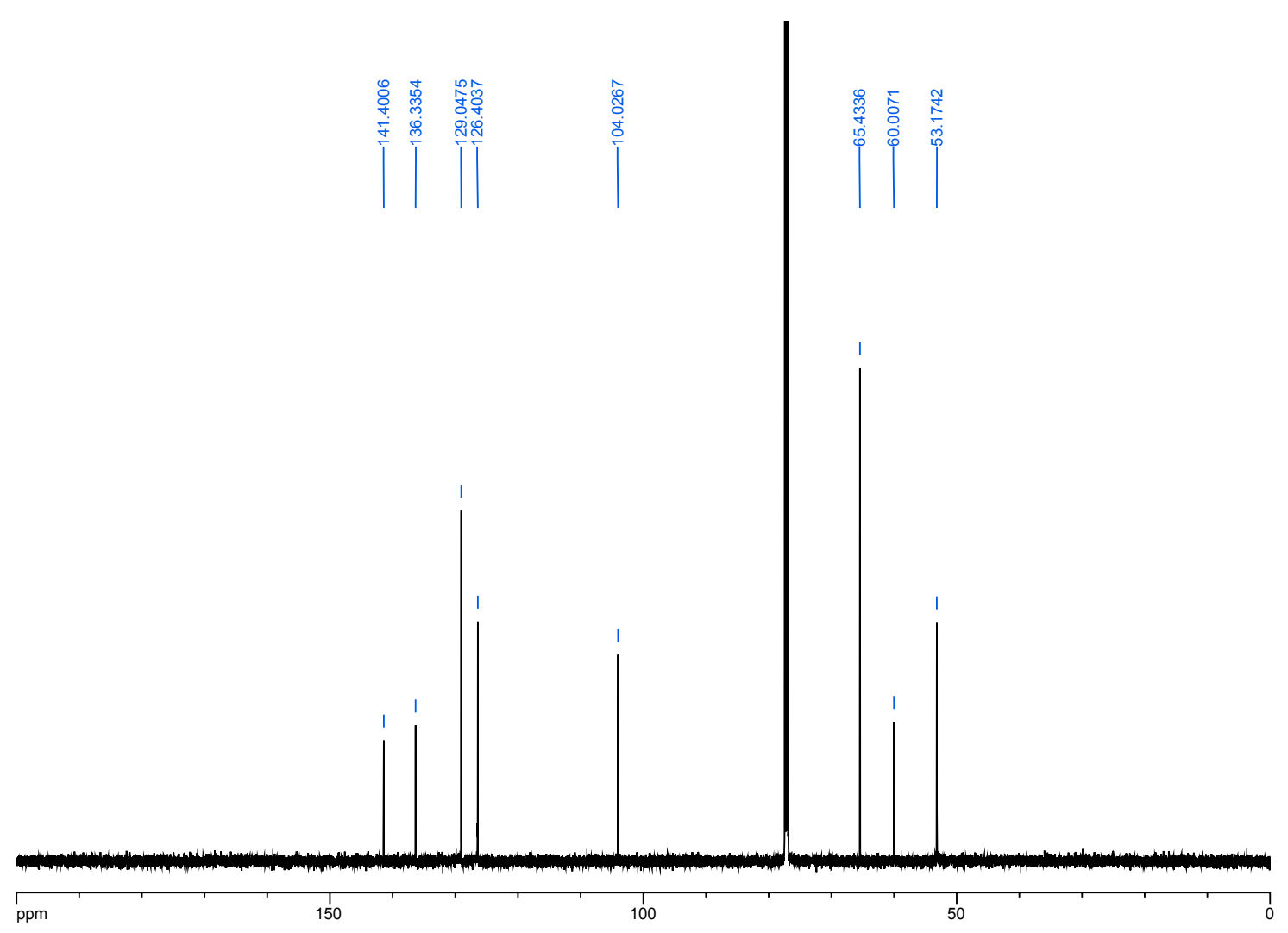


ESI-TOF MS: 2 (positive mode).

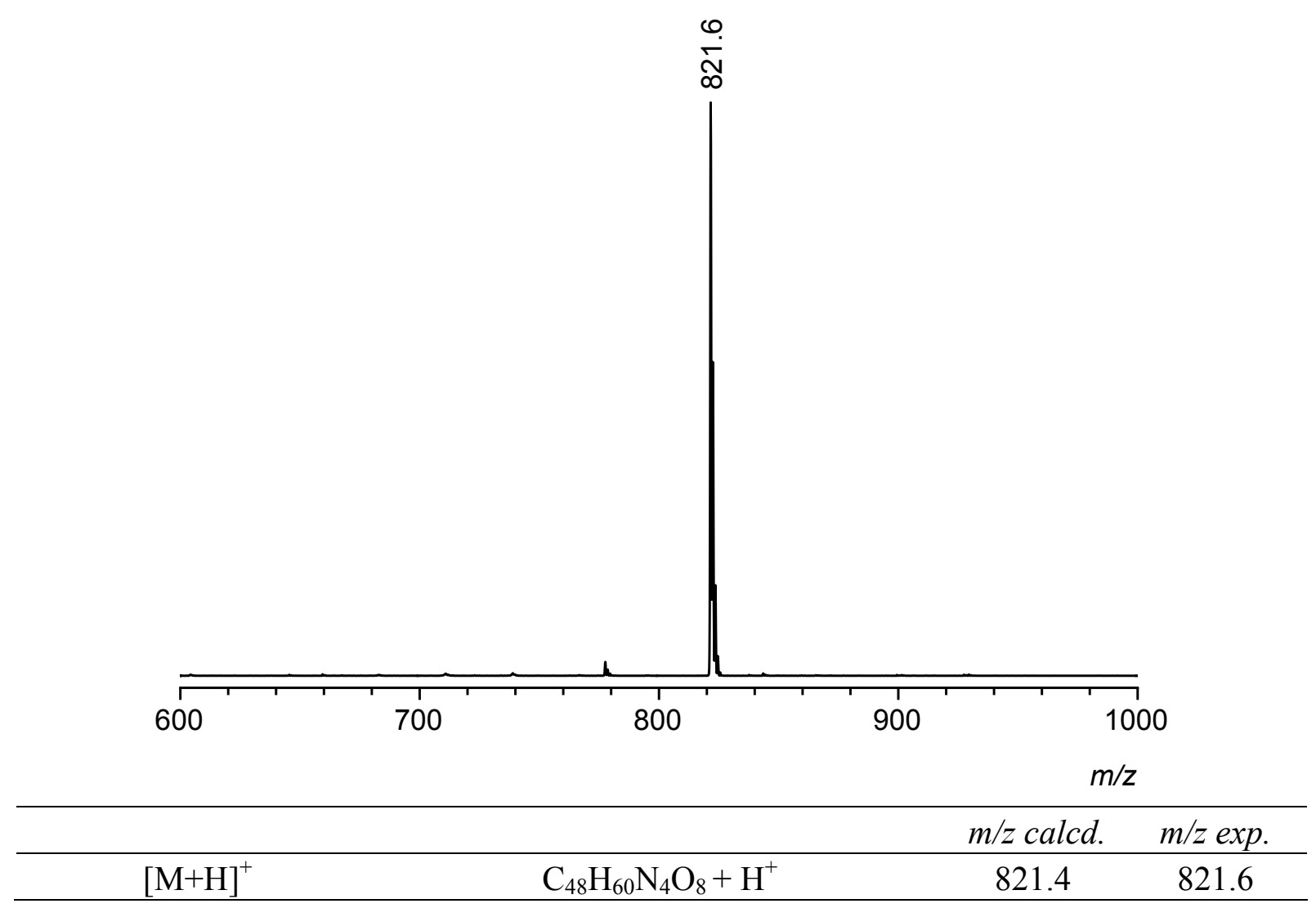


${ }^{1}$ H NMR: 3 (400 MHz, $\left.\mathrm{CDCl}_{3}, 25^{\circ} \mathrm{C}\right)$.

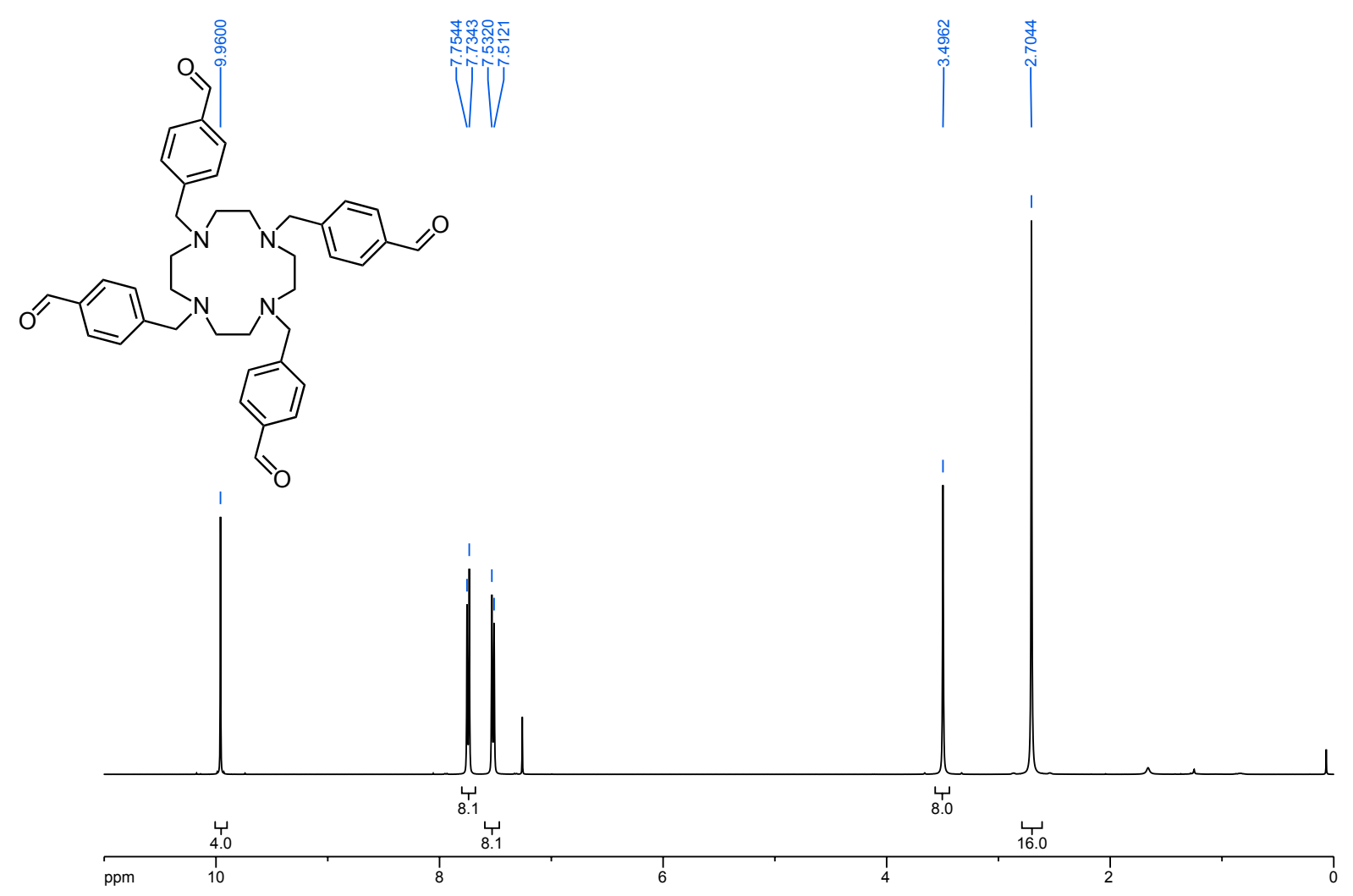

${ }^{13} \mathrm{C}$ NMR: 3 (101 MHz, $\left.\mathrm{CDCl}_{3}, 25^{\circ} \mathrm{C}\right)$.

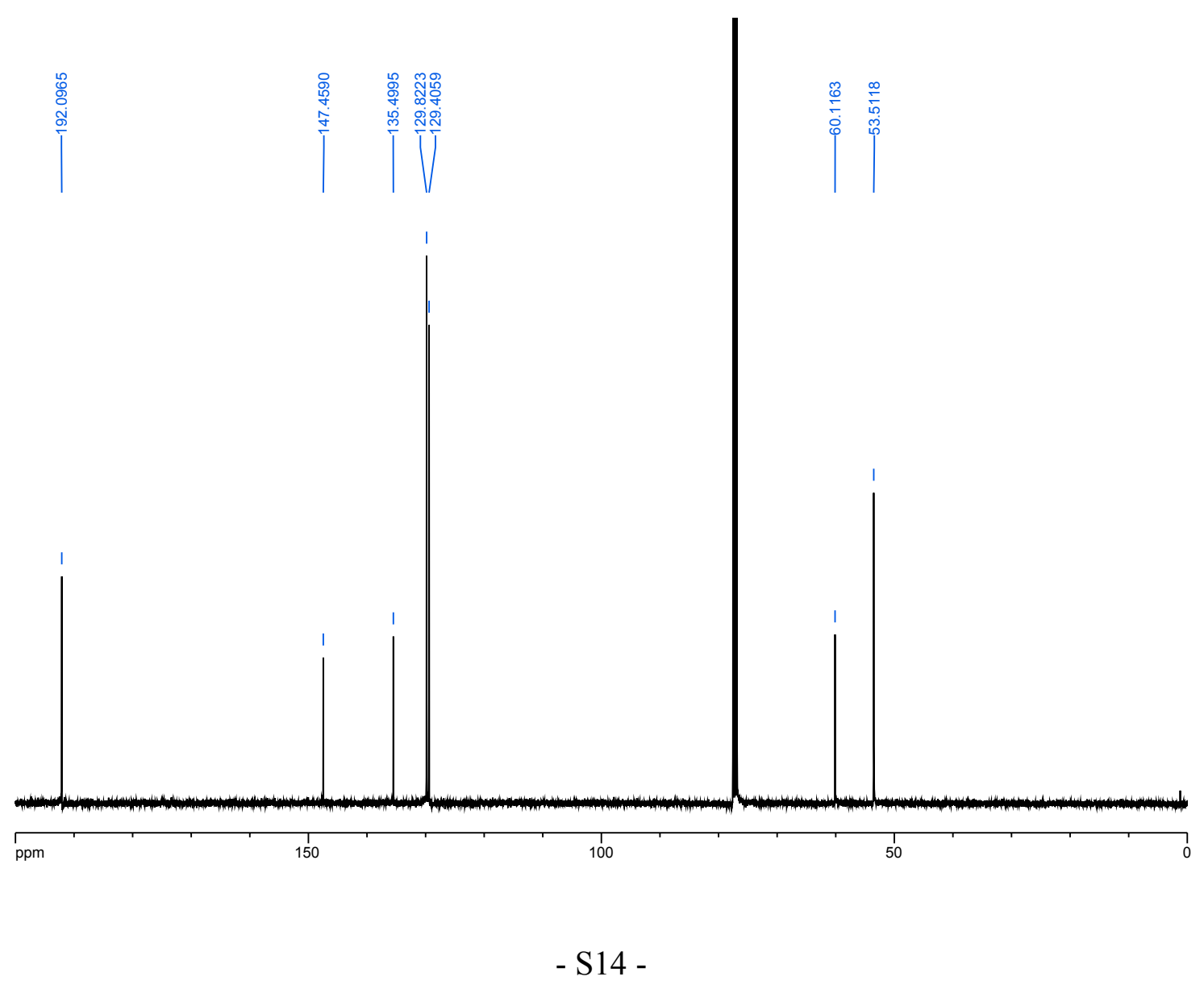


ESI-TOF MS: 3 (positive mode).

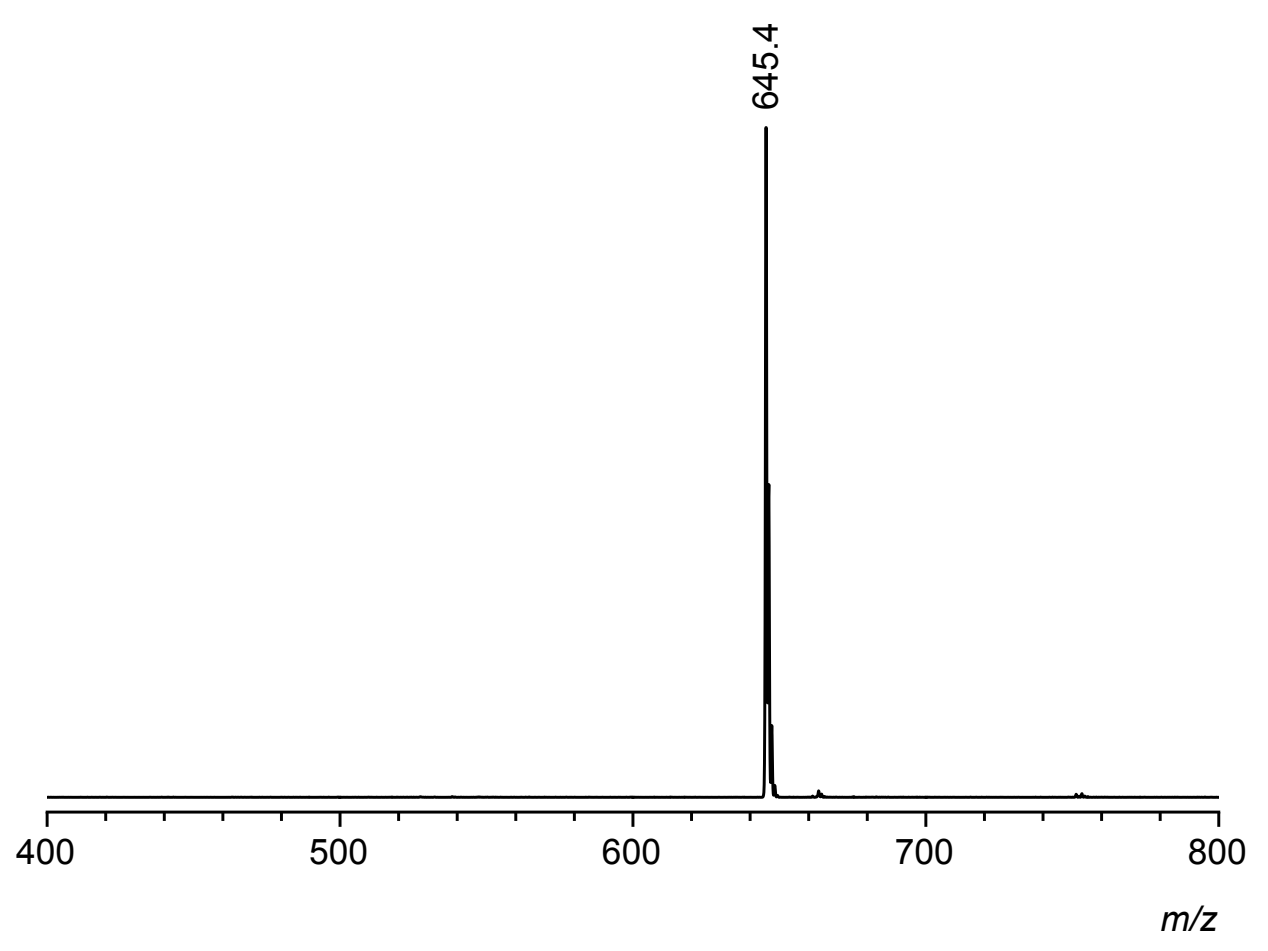

$m / z$ calcd. $\quad m / z \exp$.

$\begin{array}{llll}{[\mathrm{M}+\mathrm{H}]^{+}} & \mathrm{C}_{40} \mathrm{H}_{44} \mathrm{~N}_{4} \mathrm{O}_{4}+\mathrm{H}^{+} & 645.3 & 645.4\end{array}$


${ }^{1}$ H NMR: $5\left(400 \mathrm{MHz}, \mathrm{CDCl}_{3}, 25^{\circ} \mathrm{C}\right)$.

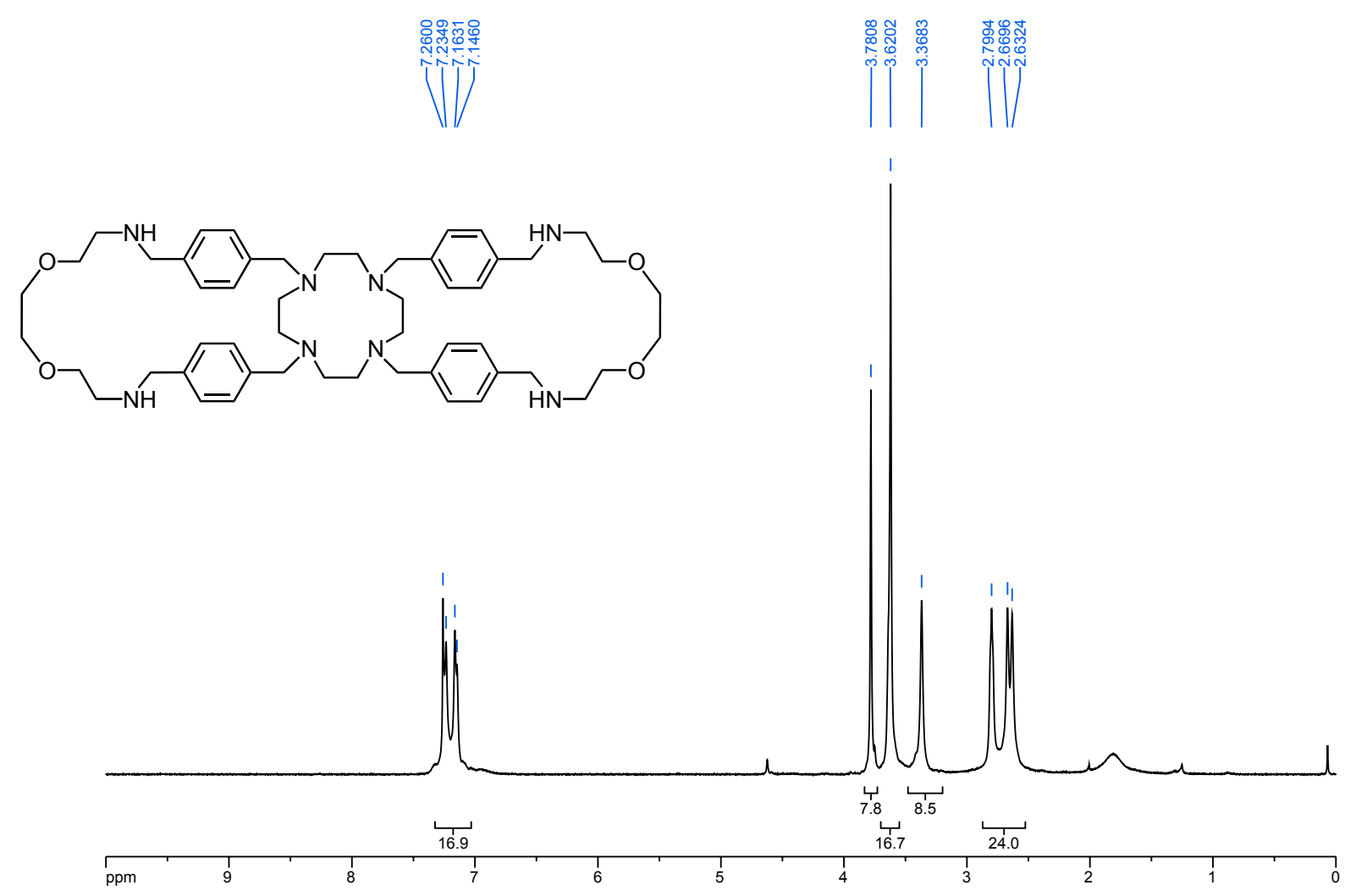

${ }^{13} \mathrm{C}$ NMR: 5 (101 MHz, $\left.\mathrm{CDCl}_{3}, 25^{\circ} \mathrm{C}\right)$.

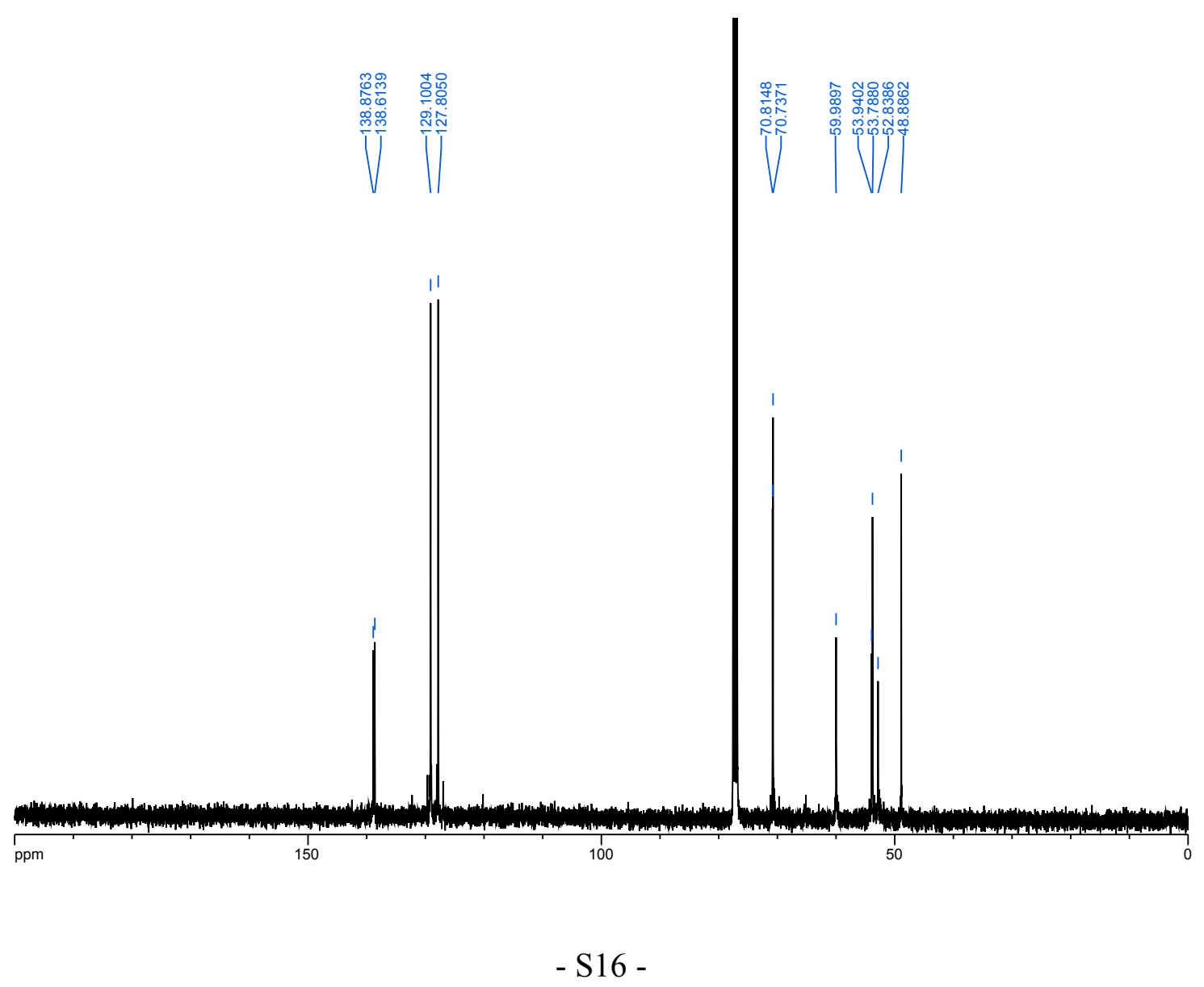


ESI-TOF MS: 5 (positive mode).

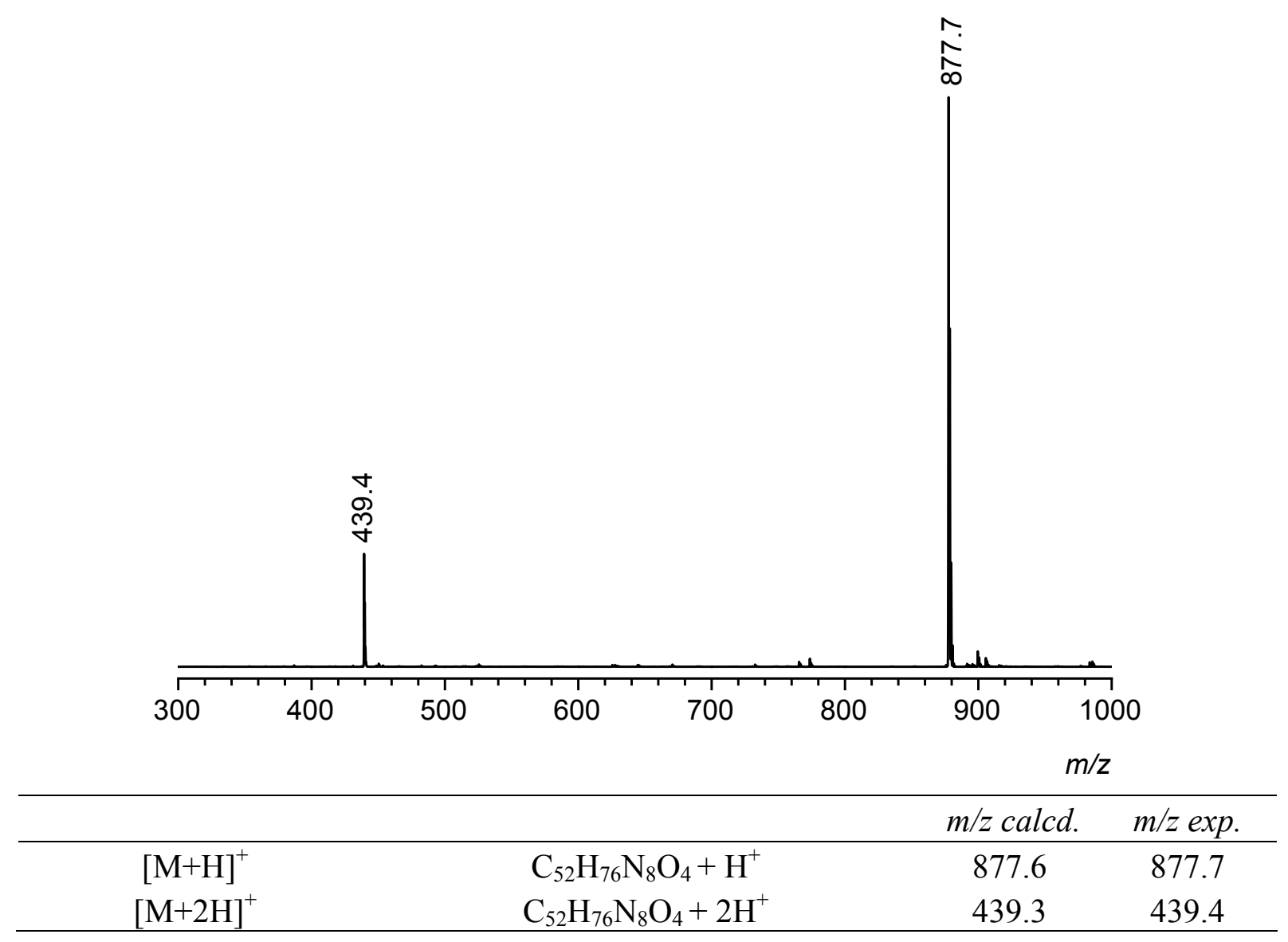


${ }^{1} \mathrm{H}$ NMR: $\mathbf{3} \cdot \mathbf{Z n C l}_{2}\left(400 \mathrm{MHz}, \mathrm{DMSO}-d_{6}, 25{ }^{\circ} \mathrm{C}\right)$.

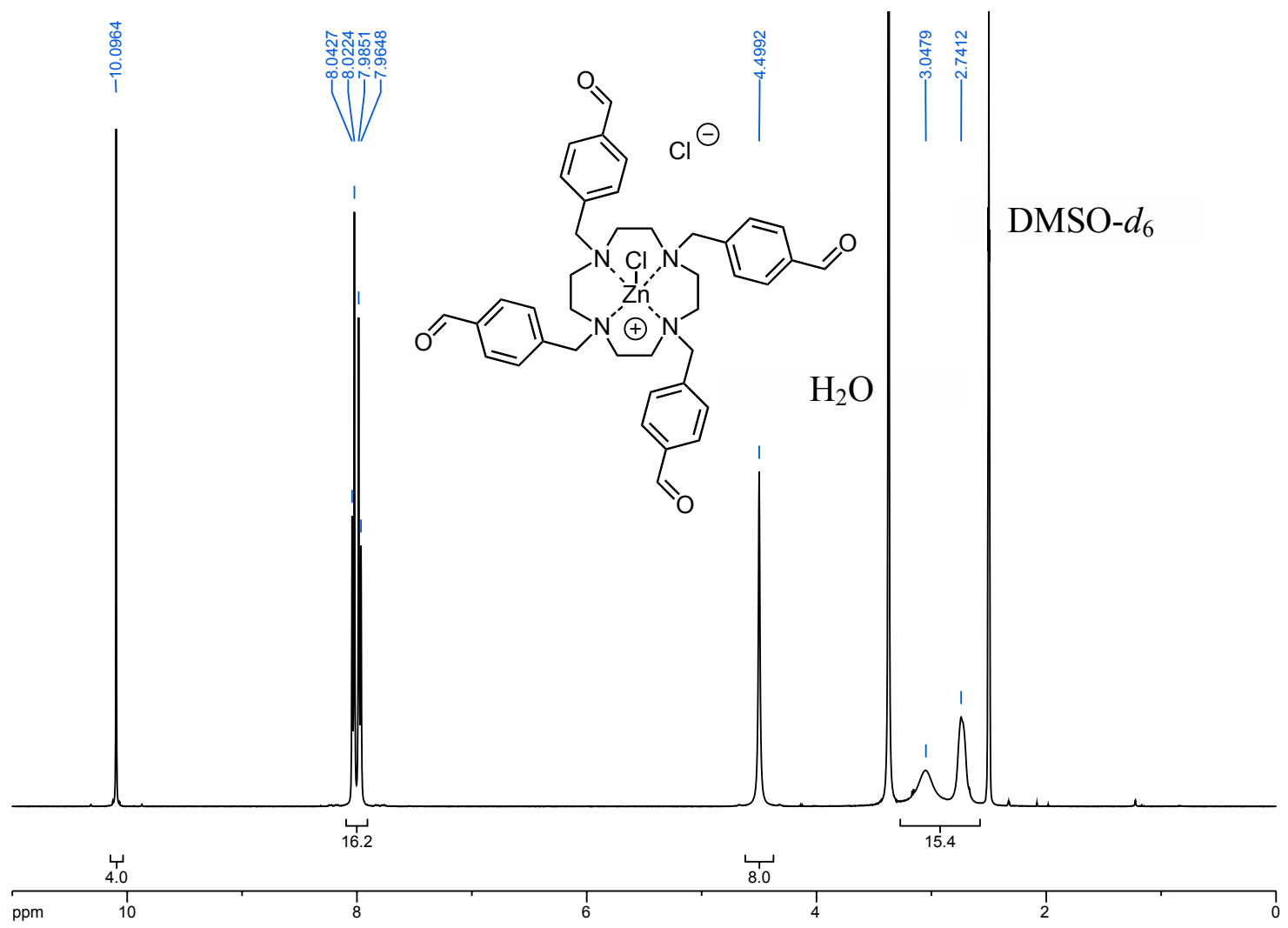

${ }^{13} \mathrm{C}$ NMR: $\mathbf{3} \cdot \mathbf{Z n C l}_{\mathbf{2}}\left(101 \mathrm{MHz}, \mathrm{DMSO}-d_{6}, 25^{\circ} \mathrm{C}\right)$.
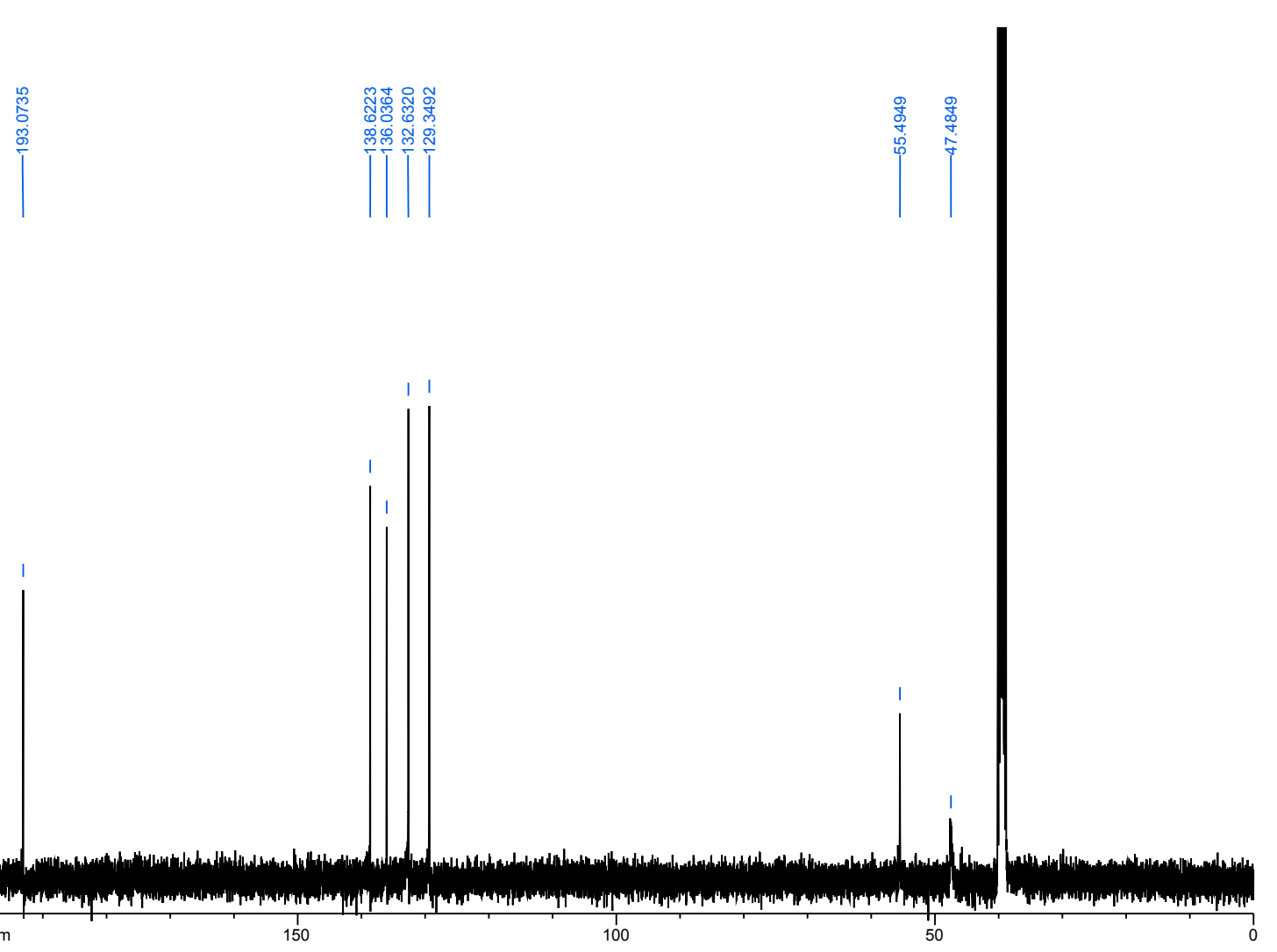

- S18 - 
ESI-TOF MS: $\mathbf{3} \cdot \mathbf{Z n C l}_{\mathbf{2}}$ (positive mode).

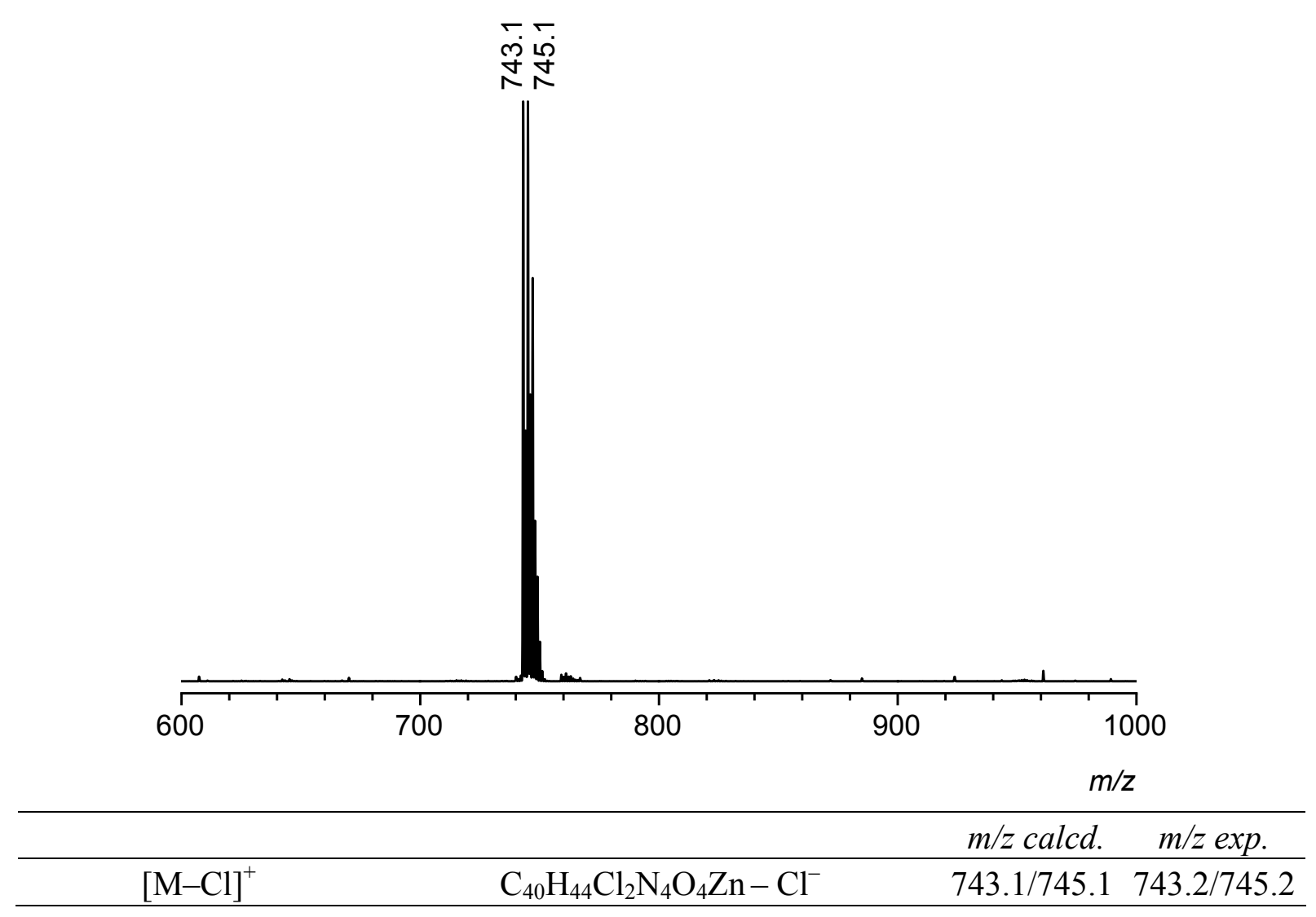


${ }^{1} \mathrm{H}$ NMR: 6. $\left(\mathrm{ZnCl}_{2}\right)_{2}\left(400 \mathrm{MHz}\right.$, DMSO- $\left.d_{6}, 25^{\circ} \mathrm{C}\right)$.
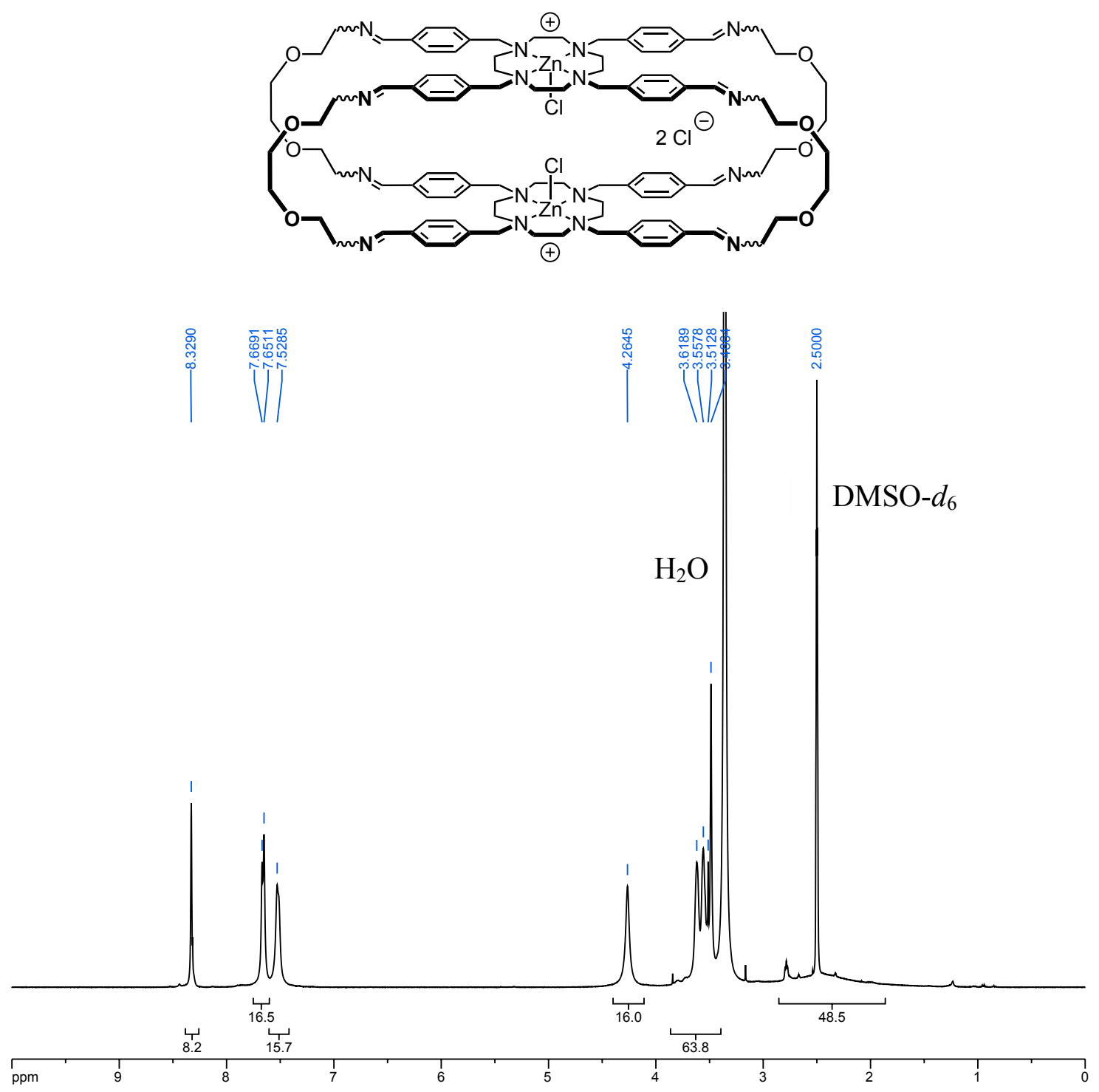
ESI-TOF MS: $6 \cdot\left(\mathrm{ZnCl}_{2}\right)_{2}$ (positive mode).

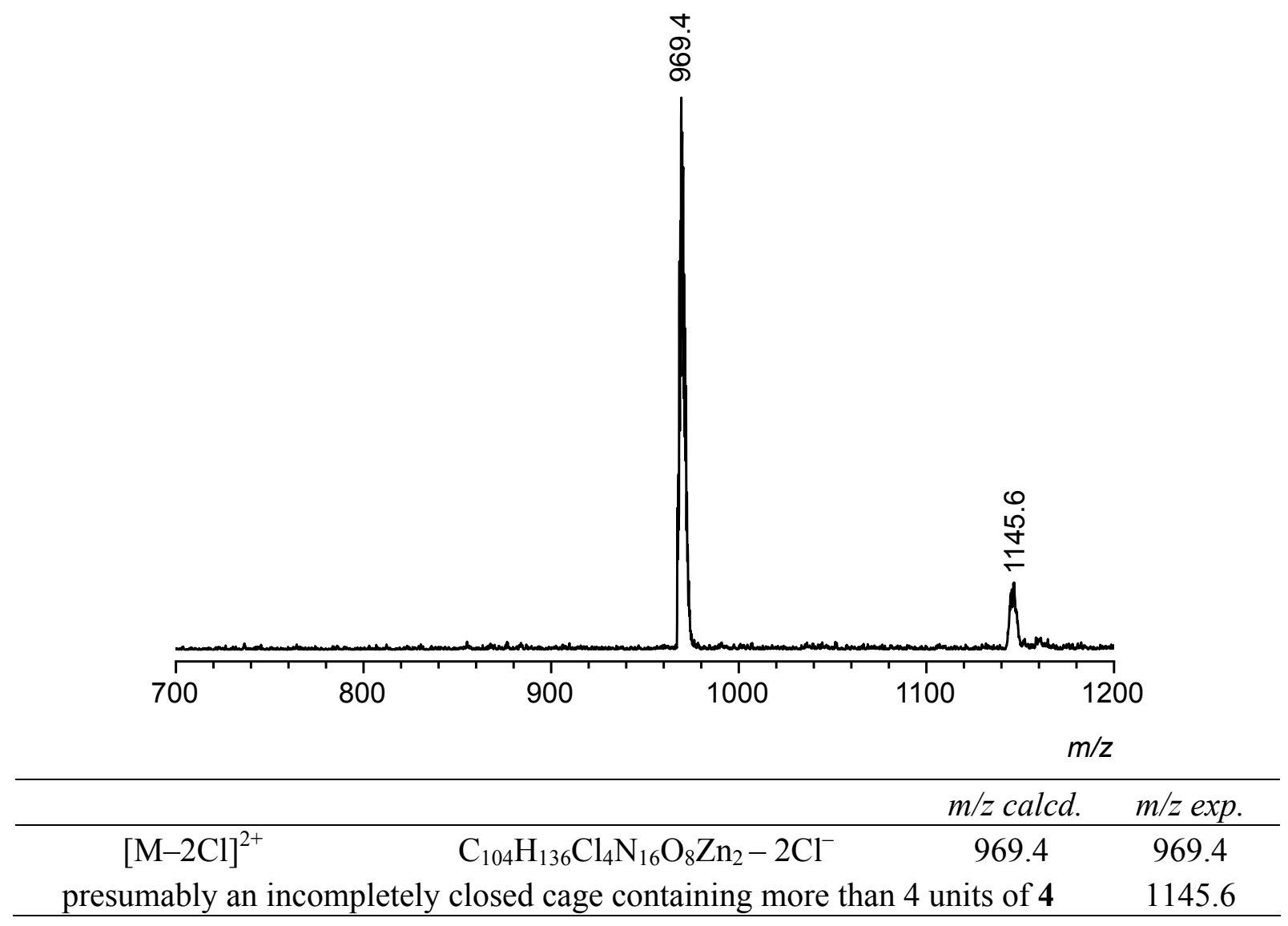


${ }^{1} \mathrm{H}$ NMR: Tris(cyclen) $\cdot\left(\mathrm{ZnCl}_{2}\right)_{3}$ formed with 1,2-diaminoethane (imine form) (400 MHz, DMSO- $d_{6}$, $25^{\circ} \mathrm{C}$ ).
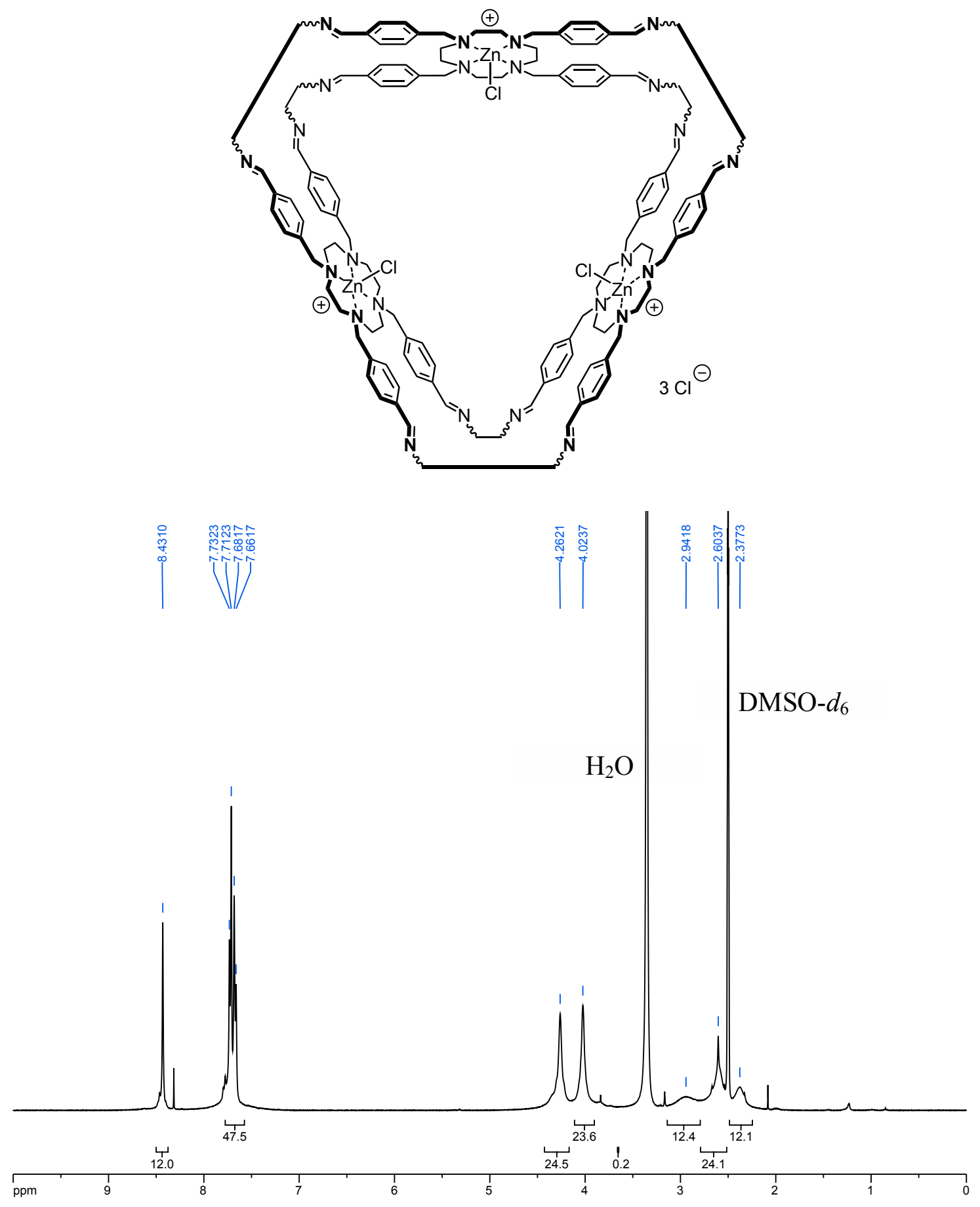
ESI-TOF MS: Tris(cyclen) $\left(\mathrm{ZnCl}_{2}\right)_{3}$ formed with 1,2-diaminoethane (imine form) (positive mode).

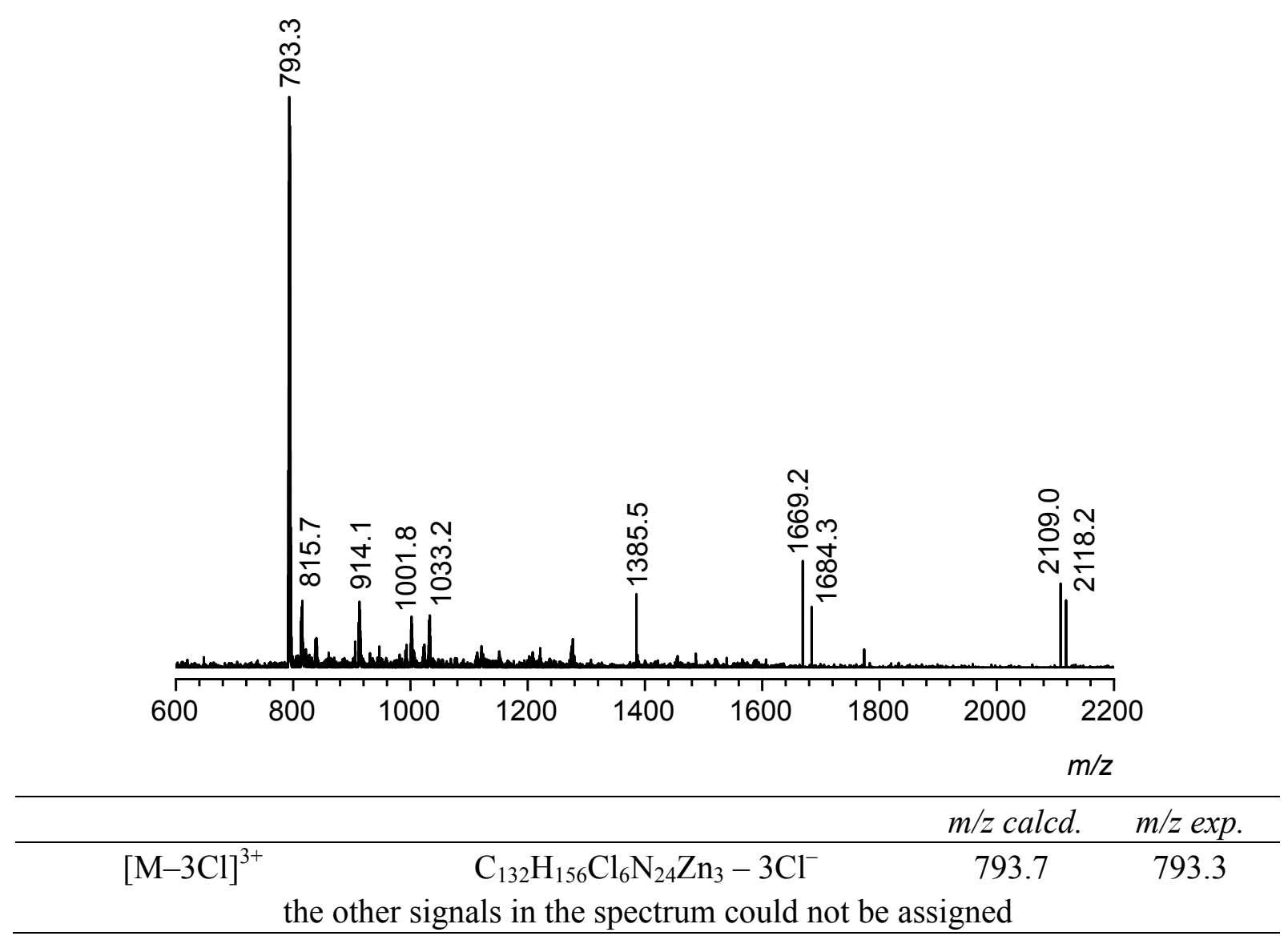


${ }^{1} \mathrm{H}$ NMR: 7·( $\left.\mathrm{ZnCl}_{2}\right)_{2}\left(400 \mathrm{MHz}\right.$, DMSO- $\left.d_{6}, 25^{\circ} \mathrm{C}\right)$.
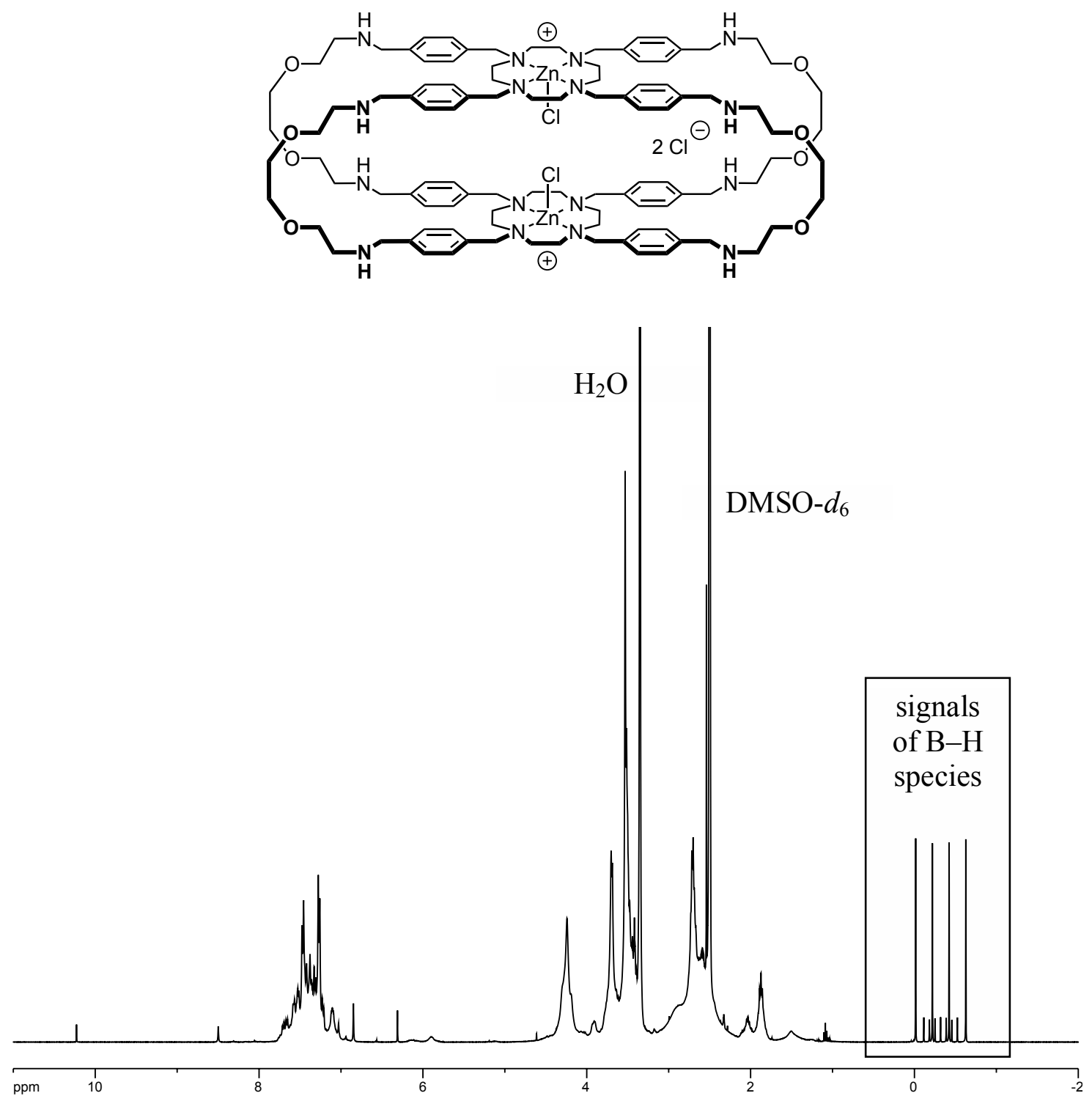
ESI-TOF MS: 7·( $\left(\mathrm{ZCl}_{2}\right)_{2}$ (positive mode).

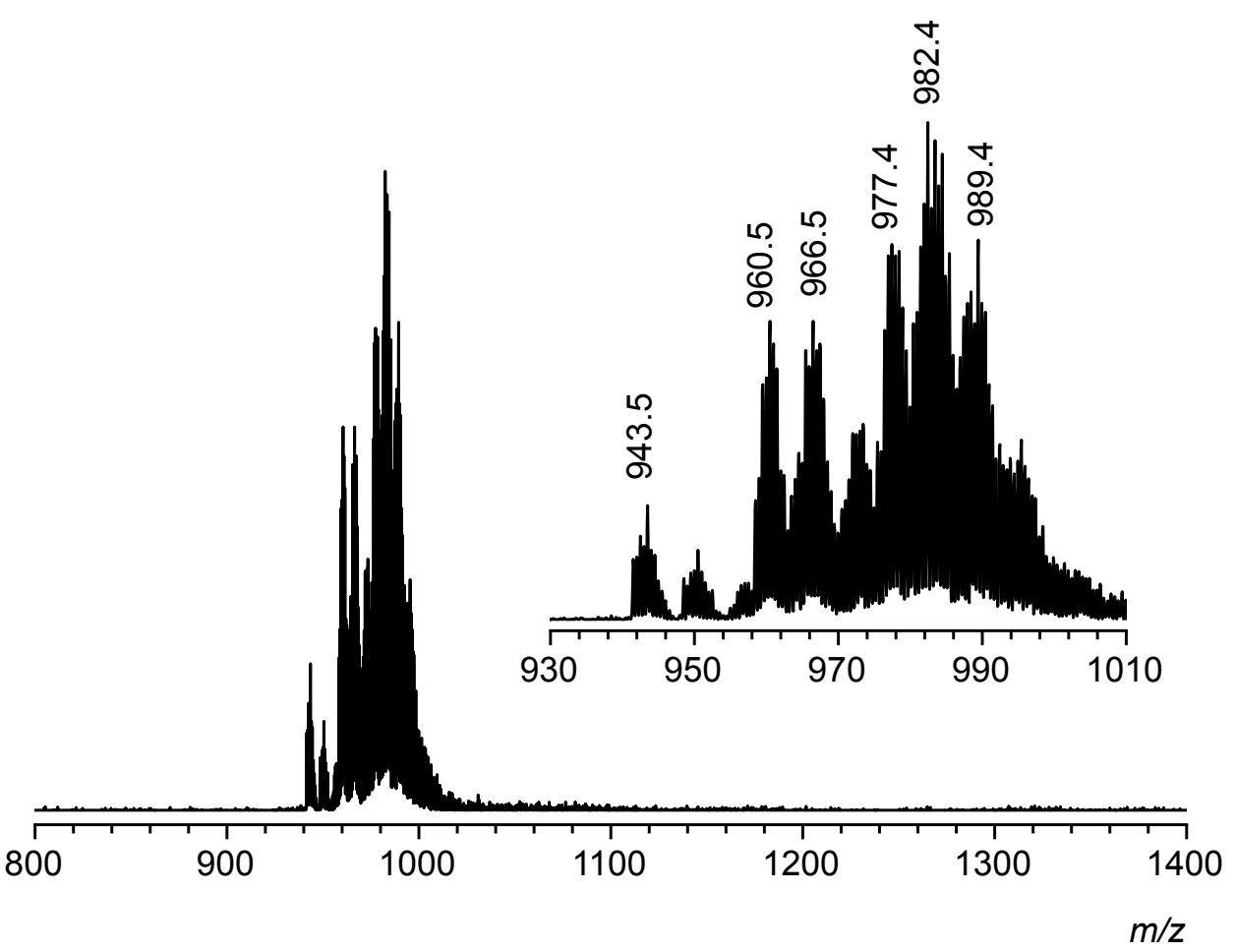

\begin{tabular}{cccc}
\hline & & $m / z$ calcd. & m/z exp. \\
\hline$\left[\mathrm{M}-4 \mathrm{Cl}+2 \mathrm{H}^{-}\right]^{2+}$ & $\mathrm{C}_{104} \mathrm{H}_{152} \mathrm{Cl}_{4} \mathrm{~N}_{16} \mathrm{O}_{8} \mathrm{Zn}_{2}-4 \mathrm{Cl}^{-}+2 \mathrm{H}^{-}$ & 943.0 & 943.5 \\
{$\left[\mathrm{M}-3 \mathrm{Cl}+\mathrm{H}^{-}\right]^{2+}$} & $\mathrm{C}_{104} \mathrm{H}_{152} \mathrm{Cl}_{4} \mathrm{~N}_{16} \mathrm{O}_{8} \mathrm{Zn}_{2}-3 \mathrm{Cl}^{-}+\mathrm{H}^{-}$ & 961.0 & 960.5 \\
{$\left[\mathrm{M}-3 \mathrm{Cl}+\mathrm{H}^{-}+\mathrm{BH}\right]^{2+}$} & $\mathrm{C}_{104} \mathrm{H}_{152} \mathrm{Cl}_{4} \mathrm{~N}_{16} \mathrm{O}_{8} \mathrm{Zn}_{2}-3 \mathrm{Cl}^{-}+\mathrm{H}^{-}+\mathrm{BH}$ & 966.0 & 966.5 \\
{$[\mathrm{M}-2 \mathrm{Cl}]^{2+}$} & $\mathrm{C}_{104} \mathrm{H}_{152} \mathrm{Cl}_{4} \mathrm{~N}_{16} \mathrm{O}_{8} \mathrm{Zn}_{2}-2 \mathrm{Cl}^{-}$ & 978.0 & 977.4 \\
{$[\mathrm{M}-2 \mathrm{Cl}+\mathrm{BH}]^{2+}$} & $\mathrm{C}_{104} \mathrm{H}_{152} \mathrm{Cl}_{4} \mathrm{~N}_{16} \mathrm{O}_{8} \mathrm{Zn}_{2}-2 \mathrm{Cl}^{-}+\mathrm{BH}$ & 983.0 & 982.4 \\
{$[\mathrm{M}-2 \mathrm{Cl}+2 \mathrm{BH}]^{2+}$} & $\mathrm{C}_{104} \mathrm{H}_{152} \mathrm{Cl}_{4} \mathrm{~N}_{16} \mathrm{O}_{8} \mathrm{Zn}_{2}-2 \mathrm{Cl}^{-}+2 \mathrm{BH}$ & 989.5 & 989.4 \\
\hline
\end{tabular}

The mass spectrum indicates the presence of borane adducts, presumably to the nitrogen atoms. 
${ }^{1} \mathrm{H}$ NMR: $7\left(400 \mathrm{MHz}, \mathrm{CDCl}_{3}, 25^{\circ} \mathrm{C}\right)$.

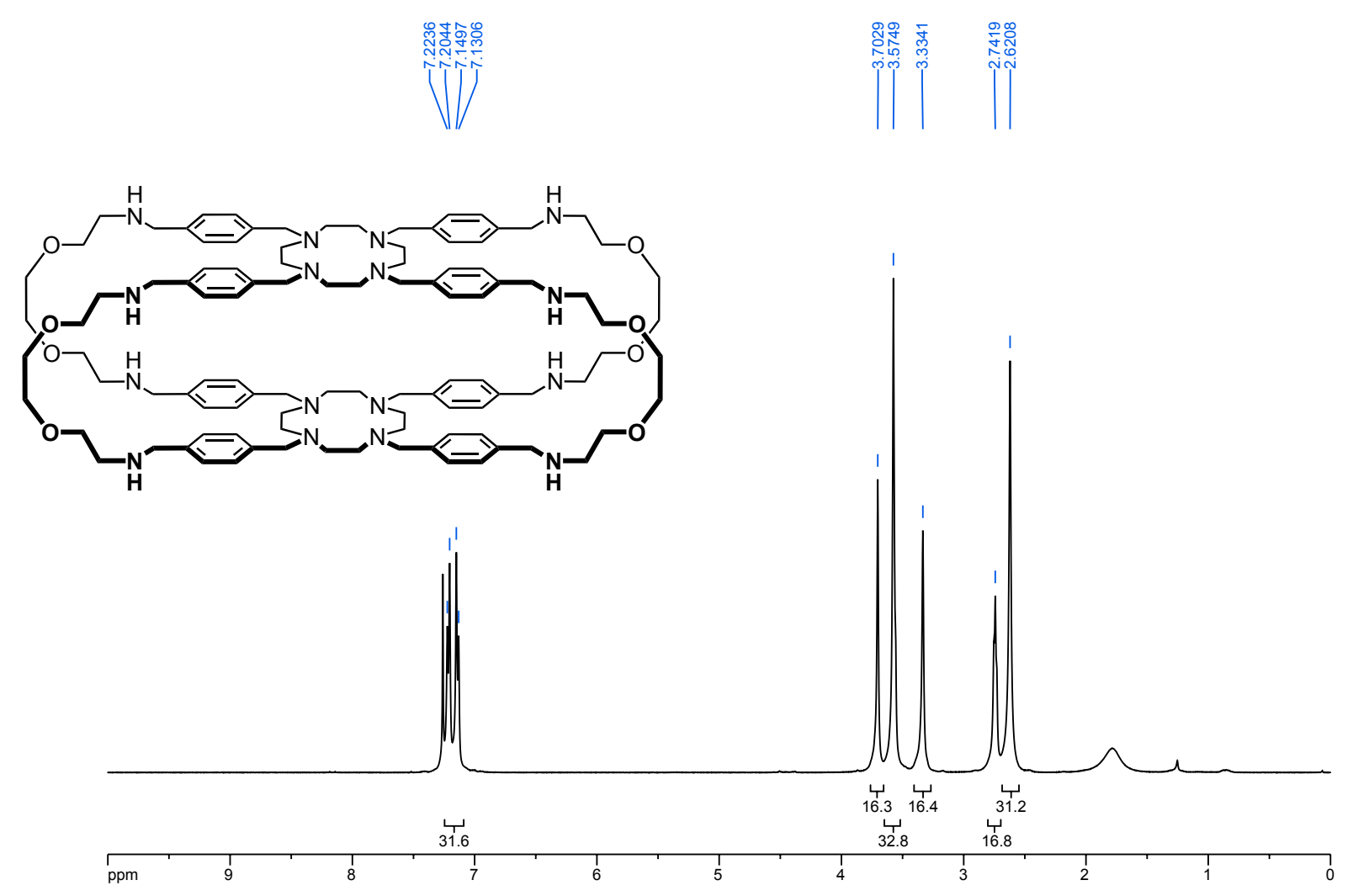

${ }^{13} \mathrm{C}$ NMR: 7 (101 MHz, $\left.\mathrm{CDCl}_{3}, 25^{\circ} \mathrm{C}\right)$.

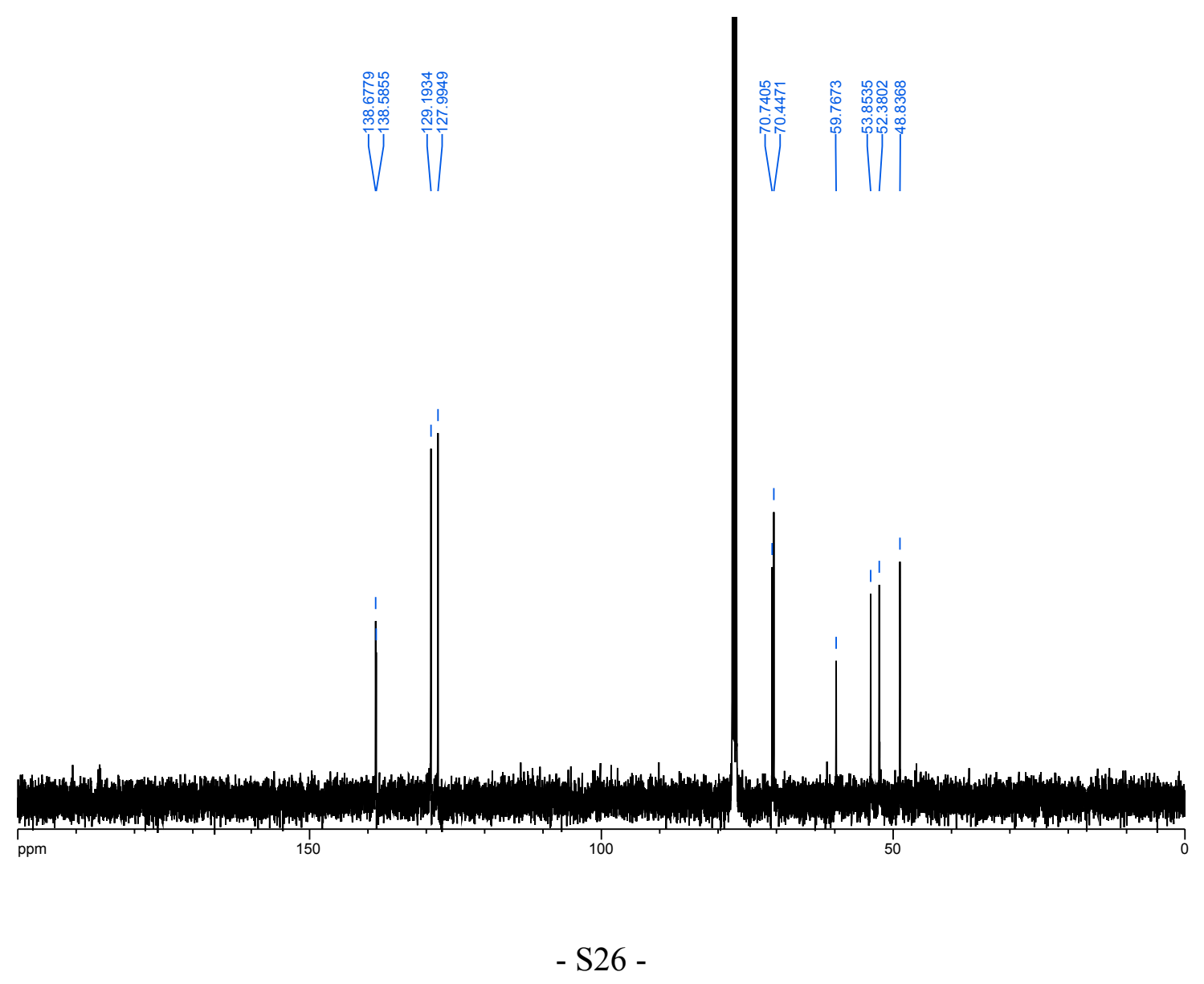


ESI-TOF MS: 7 (positive mode).

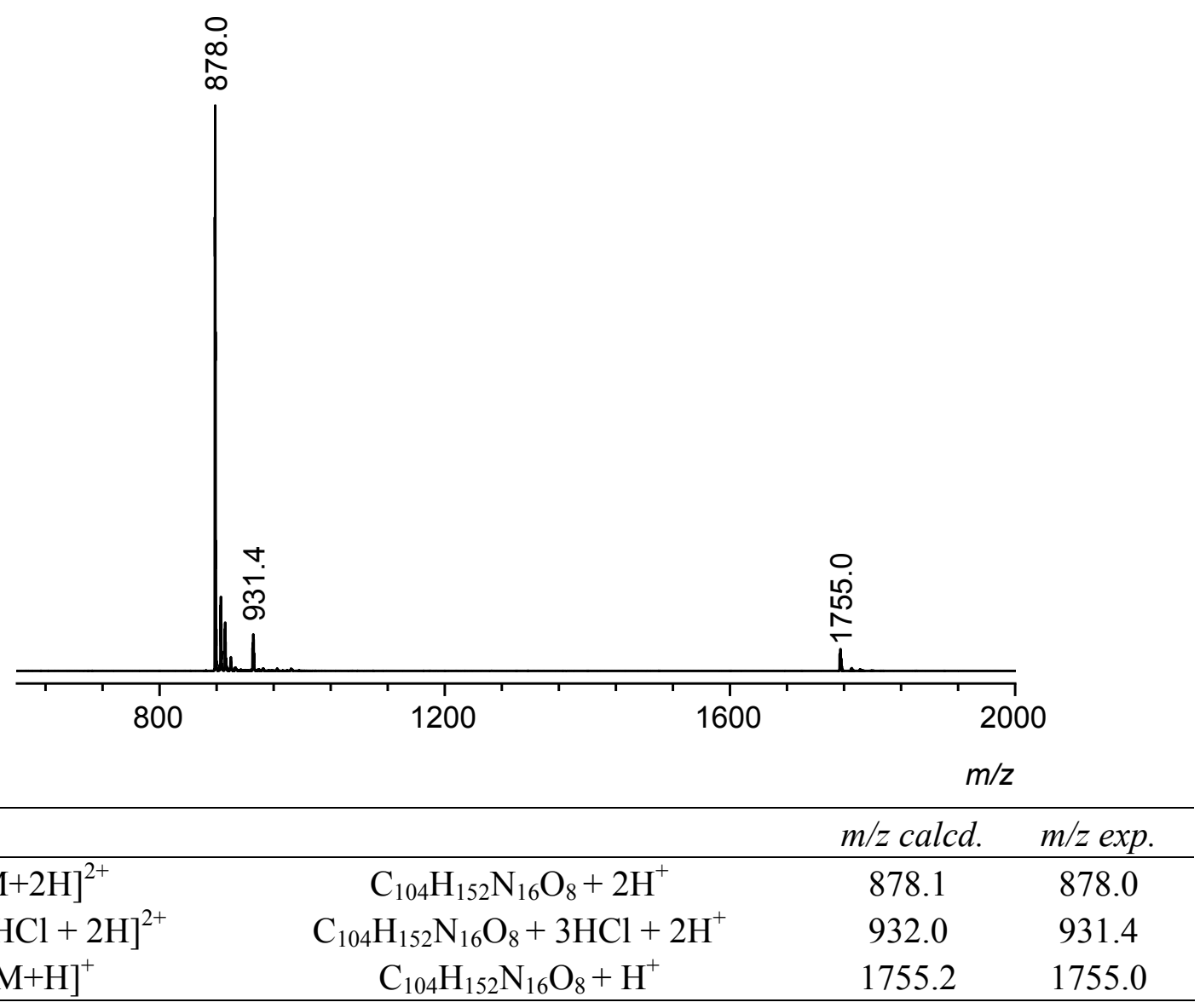

Electromotive Force Measurements

The potentiometric titrations were carried out at $298.1 \pm 0.1 \mathrm{~K}$ using $\mathrm{NaCl} 0.15 \mathrm{M}$ as supporting electrolyte. The experimental procedure (burette, potentiometer, cell, stirrer, microcomputer, etc.) has been fully described elsewhere. ${ }^{4}$ The acquisition of the emf data was performed with the computer program PASAT. ${ }^{5}$ The reference electrode was a Crison $5240 \mathrm{Ag} / \mathrm{AgCl}$ electrode in 0.5 $\mathrm{M} \mathrm{NaCl}$ solution. A Wilhelm bridge filled with $0.5 \mathrm{M} \mathrm{NaCl}$ was used to separate the glass and the reference electrode. The glass electrode (Crison $5250 \mathrm{Ag} / \mathrm{AgCl}$ ) was calibrated as a hydrogen-ion concentration probe by titration of previously standardized amounts of $\mathrm{HCl}$ with $\mathrm{CO}_{2}$-free $\mathrm{NaOH}$ solutions and determining the equivalent point by the Gran's method, ${ }^{6}$ which gives the standard potential, $\mathrm{E}^{\circ}$, and the ionic product of water $\left(\mathrm{p} K_{\mathrm{W}}=13.73(1)\right)$. The computer program HYPERQUAD $^{7}$ was used to fit the protonation and stability constants. ${ }^{8,9}$ 


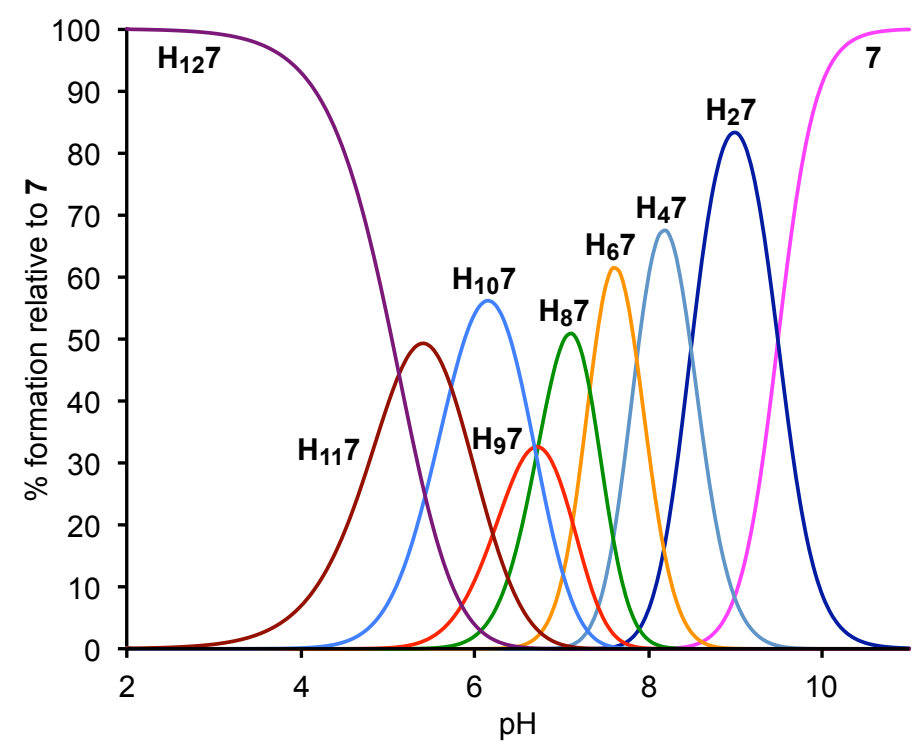

Figure S3: Speciation diagram for the protonation of $7(0.15 \mathrm{M} \mathrm{NaCl}, \mathrm{T}=298.1 \pm 0.1 \mathrm{~K})$.

Table S1: Stability Constants for the System Cu(II)-7, determined in $0.15 \mathrm{M} \mathrm{NaCl}$ at $298.1 \mathrm{~K}$.

\begin{tabular}{cc}
\hline Reaction $^{\mathrm{a}}$ & $\log K$ \\
\hline $\mathrm{Cu}+10 \mathrm{H}+7 \rightleftharpoons \mathrm{CuH}_{10} 7$ & $86.91(4)^{\mathrm{b}}$ \\
$\mathrm{Cu}+9 \mathrm{H}+7 \rightleftharpoons \mathrm{CuH}_{9} 7$ & $81.78(4)$ \\
$\mathrm{Cu}+8 \mathrm{H}+7 \rightleftharpoons \mathrm{CuH}_{8} 7$ & $75.5(5)$ \\
$\mathrm{Cu}+6 \mathrm{H}+7 \rightleftharpoons \mathrm{CuH}_{6} 7$ & $61.17(5)$ \\
$\mathrm{Cu}+4 \mathrm{H}+7 \rightleftharpoons \mathrm{CuH}_{4} 7$ & $45.49(5)$ \\
$\mathrm{Cu}+3 \mathrm{H}+7 \rightleftharpoons \mathrm{CuH}_{3} 7$ & $37.0(1)$ \\
$\mathrm{Cu}+2 \mathrm{H}+7 \rightleftharpoons \mathrm{CuH}_{2} 7$ & $28.78(7)$ \\
$\mathrm{Cu}+\mathrm{H}+7 \rightleftharpoons \mathrm{CuH}_{7}$ & $19.97(6)$ \\
$\mathrm{Cu}+7 \rightleftharpoons \mathrm{Cu}_{7}$ & $10.62(5)$ \\
$2 \mathrm{Cu}+4 \mathrm{H}+7 \rightleftharpoons \mathrm{Cu}_{2} \mathrm{H}_{4} 7$ & $49.44(5)$ \\
$2 \mathrm{Cu}+2 \mathrm{H}+7 \rightleftharpoons \mathrm{Cu}_{2} \mathrm{H}_{2} 7$ & $33.33(5)$ \\
$2 \mathrm{Cu}+7 \rightleftharpoons \mathrm{Cu}_{2} 7$ & $16.13(5)$ \\
$2 \mathrm{Cu}+\mathbf{7}+2 \mathrm{H} \mathrm{O} \rightleftharpoons \mathrm{Cu}_{2} 7(\mathrm{OH})_{2}+2 \mathrm{H}$ & $-3.22(5)$ \\
\hline
\end{tabular}

${ }^{\mathrm{a}}$ Charges omitted. ${ }^{\mathrm{b}}$ Values in parentheses are standard deviations in the last significant figure. 

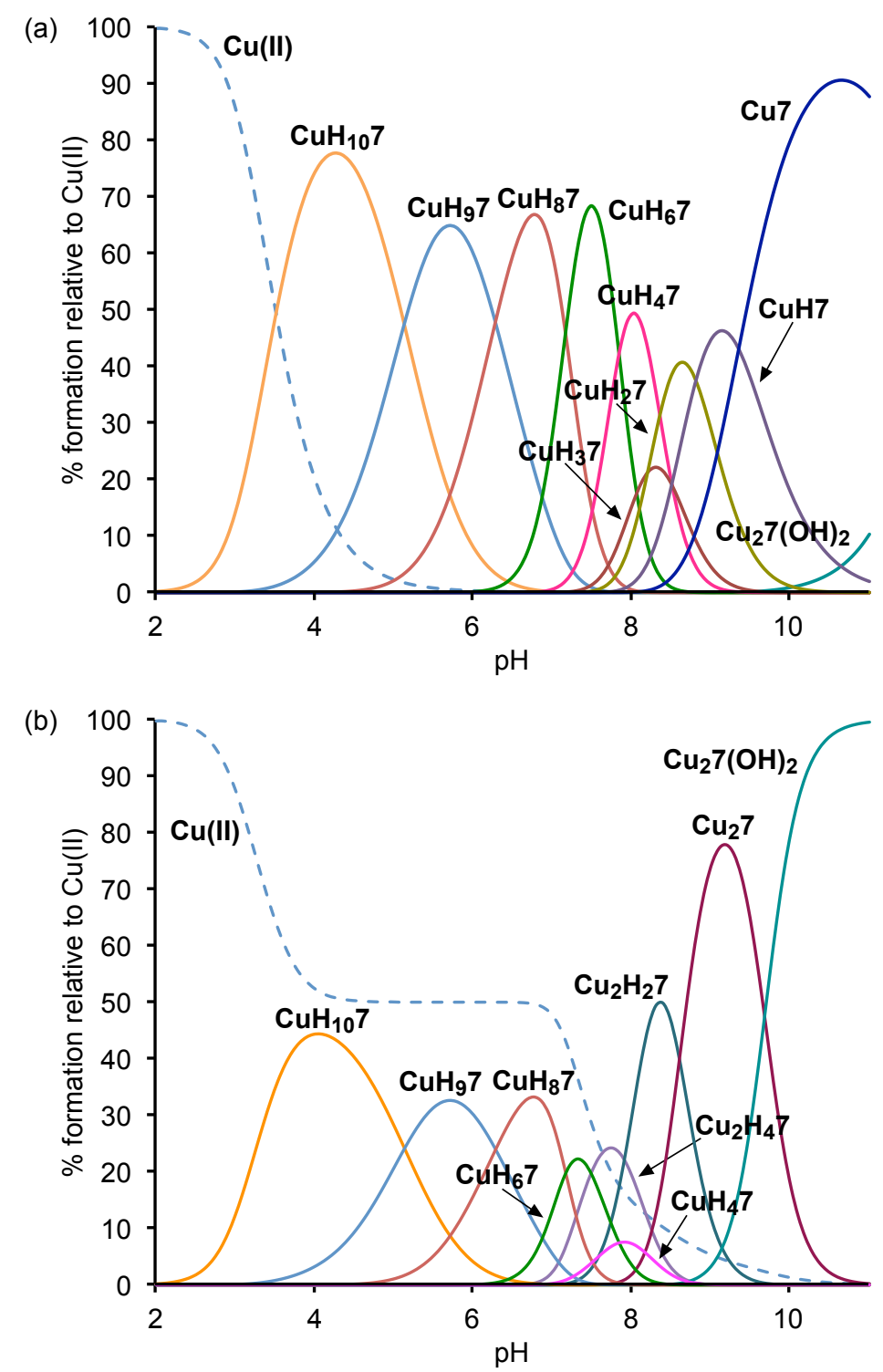

Figure S4: Plot of the distribution diagram of the system Cu(II)-7 (a) for 1:1 molar ratio ([Cu(II)] $\left.=1 \times 10^{-3} \mathrm{M}, I=0.15 \mathrm{M} \mathrm{NaCl}, \mathrm{T}=298.1 \pm 0.1 \mathrm{~K}\right) ;\left(\right.$ b) $1: 2$ molar ratio $\left([\mathrm{Cu}(\mathrm{II})]=2 \times 10^{-3} \mathrm{M}, \mathrm{I}=\right.$ $0.15 \mathrm{M} \mathrm{NaCl}, \mathrm{T}=298.1 \pm 0.1 \mathrm{~K})$. 
$\underline{\text { HR-ESI Mass Spectrometric Measurements }}$

HR-ESI mass spectra of solutions (water/methanol, $1: 1(v / v)$ ) containing 7 and $\mathrm{Cu}\left(\mathrm{ClO}_{4}\right)_{2} \cdot 6 \mathrm{H}_{2} \mathrm{O}$ in 1:1, 1:2 and 1:3 molar ratio were acquired in the positive ion mode using a Triple TOF 5600 hybrid quadrupole time-of-flight (TOF) mass spectrometer. $\mathrm{N}_{2}$ was used as a curtain and nebulizing gas. The experiments were performed at a voltage of $5300 \mathrm{~V}$ and GS1 and GS2 (35 psi) ion source gas at $723.15 \mathrm{~K}$. The AB SCIEX Peak View software was used for the analysis of the data.

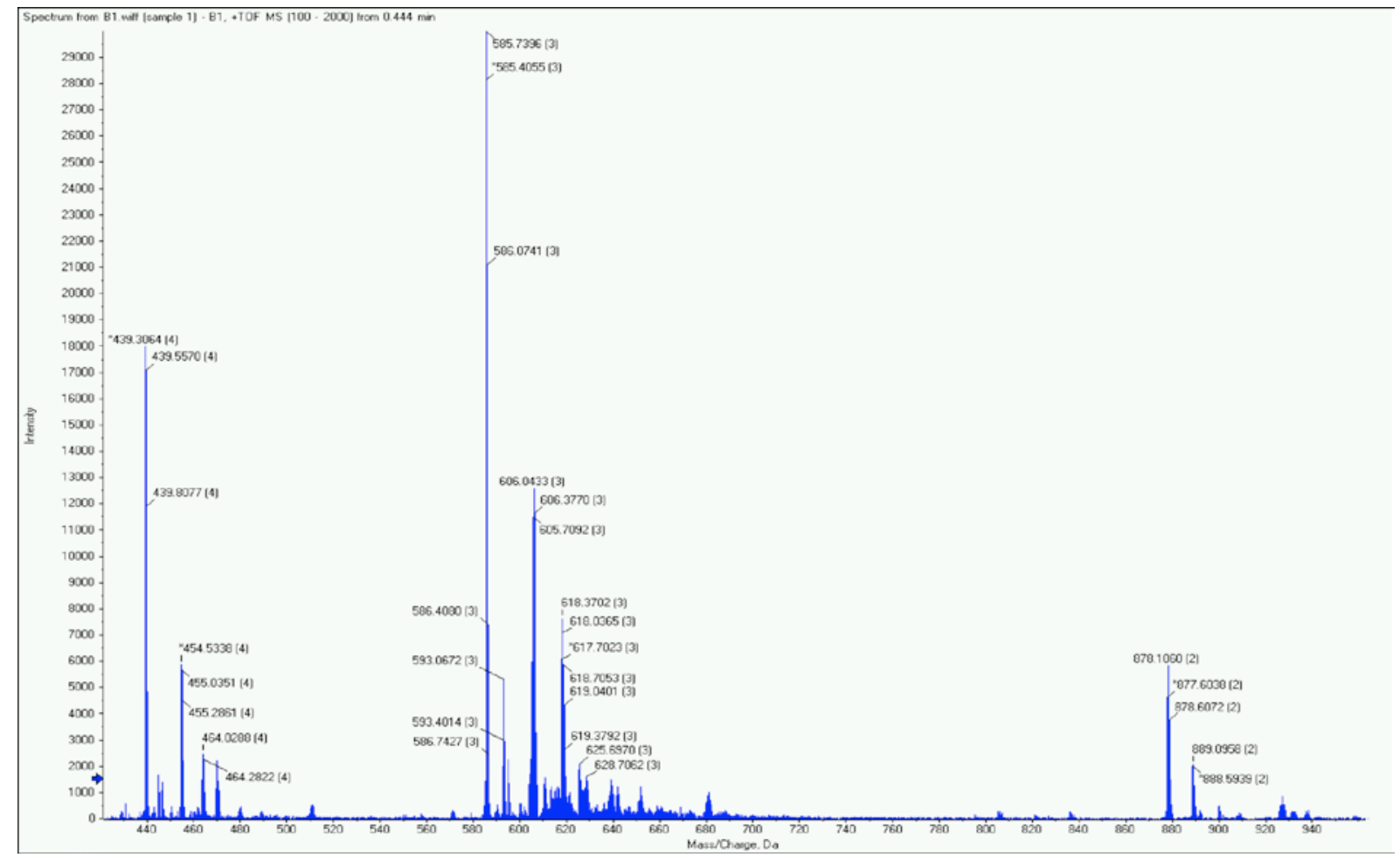

Figure S5: HR-ESI-MS spectrum of 7 in water/methanol, $1: 1(\mathrm{v} / \mathrm{v})$ containing 1 equiv of $\mathrm{Cu}\left(\mathrm{ClO}_{4}\right)_{2}$.

\begin{tabular}{ccc}
\hline & & $m / z \exp$. \\
\hline$[\mathrm{M}+2 \mathrm{H}]^{2+}$ & $\mathrm{C}_{104} \mathrm{H}_{152} \mathrm{~N}_{16} \mathrm{O}_{8}+2 \mathrm{H}^{+}$ & 878.1068 \\
{$[\mathrm{M}+3 \mathrm{H}]^{3+}$} & $\mathrm{C}_{104} \mathrm{H}_{152} \mathrm{~N}_{16} \mathrm{O}_{8}+3 \mathrm{H}^{+}$ & 585.4055 \\
{$[\mathrm{M}+4 \mathrm{H}]^{4+}$} & $\mathrm{C}_{104} \mathrm{H}_{152} \mathrm{~N}_{16} \mathrm{O}_{8}+4 \mathrm{H}^{+}$ & 439.3073 \\
{$[\mathrm{M}+\mathrm{Cu}+2 \mathrm{H}]^{4+}$} & $\mathrm{C}_{104} \mathrm{H}_{152} \mathrm{~N}_{16} \mathrm{O}_{8}+\mathrm{Cu}^{2+}+2 \mathrm{H}^{+}$ & 454.5339 \\
{$[\mathrm{M}+\mathrm{Cu}+\mathrm{H}]^{3+}$} & $\mathrm{C}_{104} \mathrm{H}_{152} \mathrm{~N}_{16} \mathrm{O}_{8}+\mathrm{Cu}^{2+}+\mathrm{H}^{+}$ & 605.7092 \\
\hline
\end{tabular}



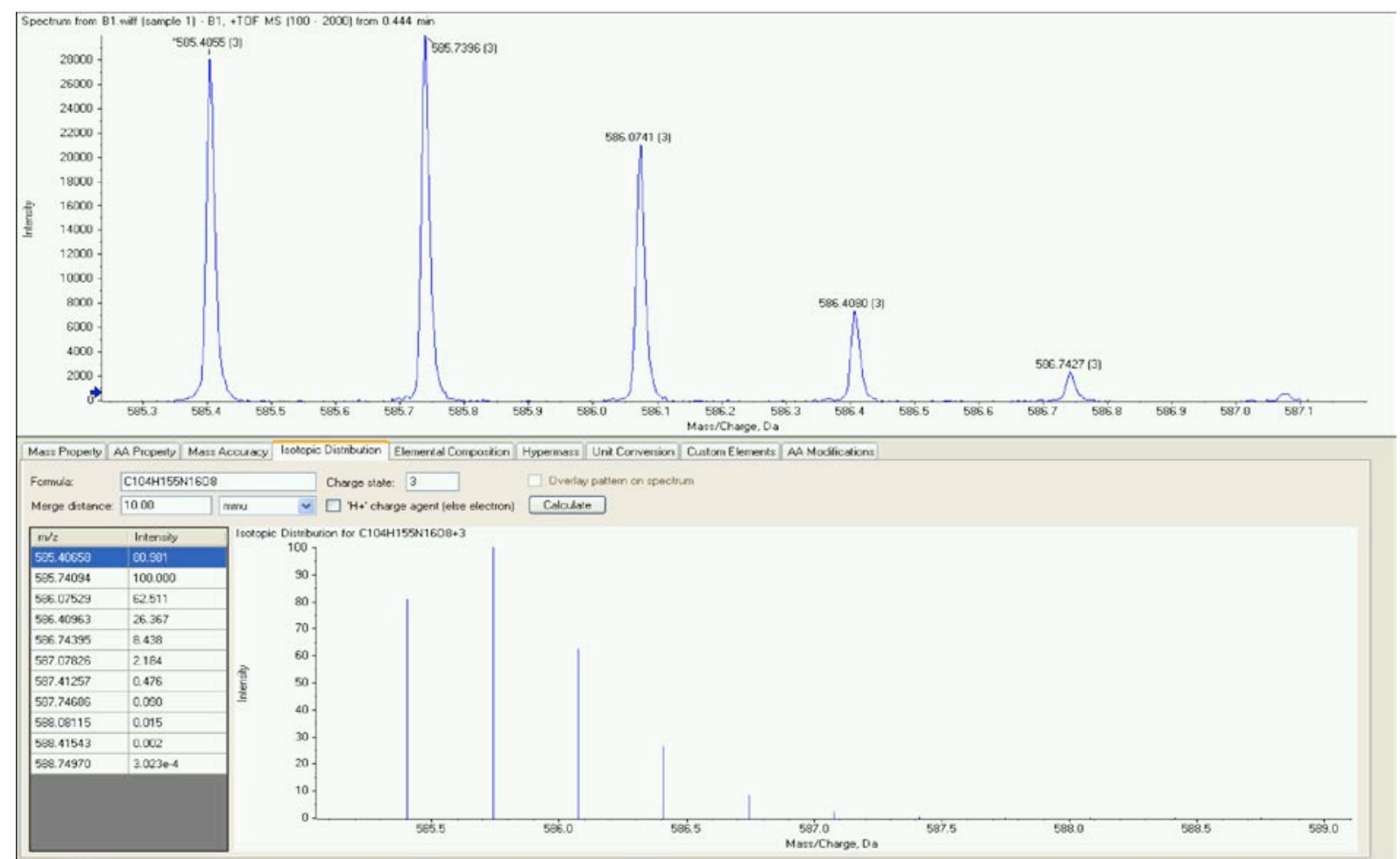

Figure S6: Experimental (top) and simulated (bottom) peaks for $\mathrm{H}_{3} 7^{3+}$.
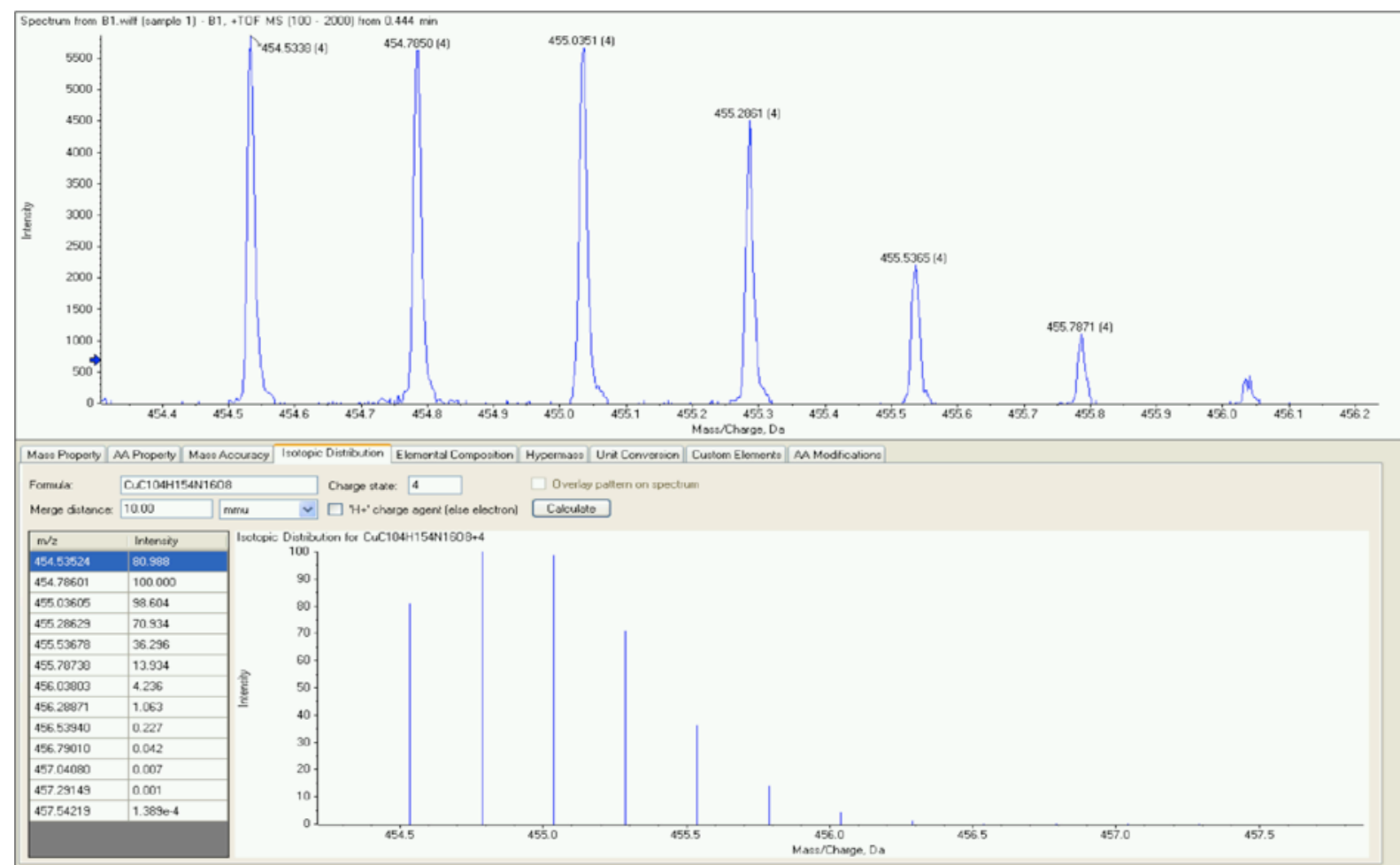

Figure S7: Experimental (top) and simulated (bottom) peaks for $\left[\mathrm{Cu}\left(\mathrm{H}_{2} 7\right)\right]^{4+}$. 


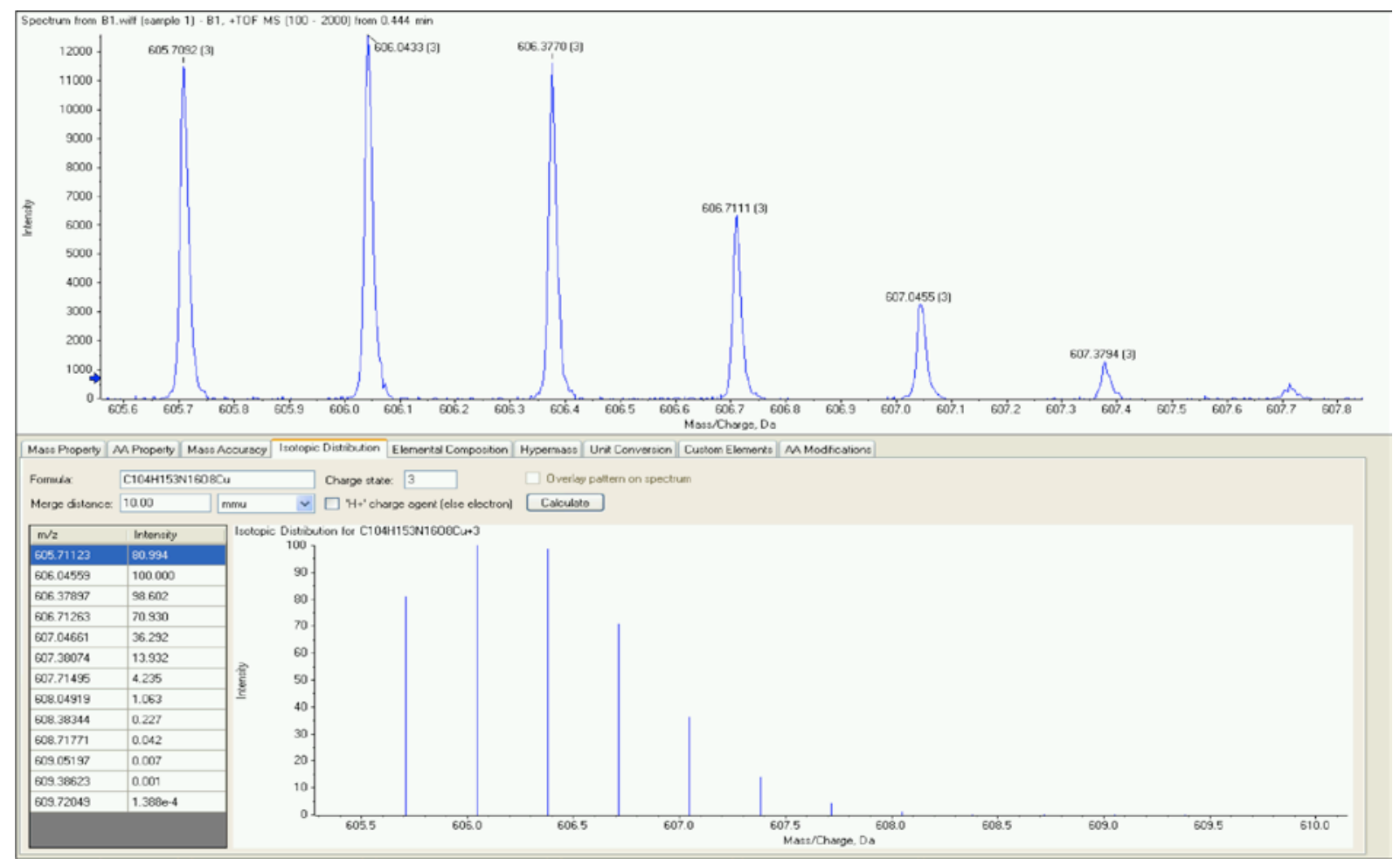

Figure S8: Experimental (top) and simulated (bottom) peaks for $[\mathrm{Cu}(\mathrm{H} 7)]^{3+}$. 


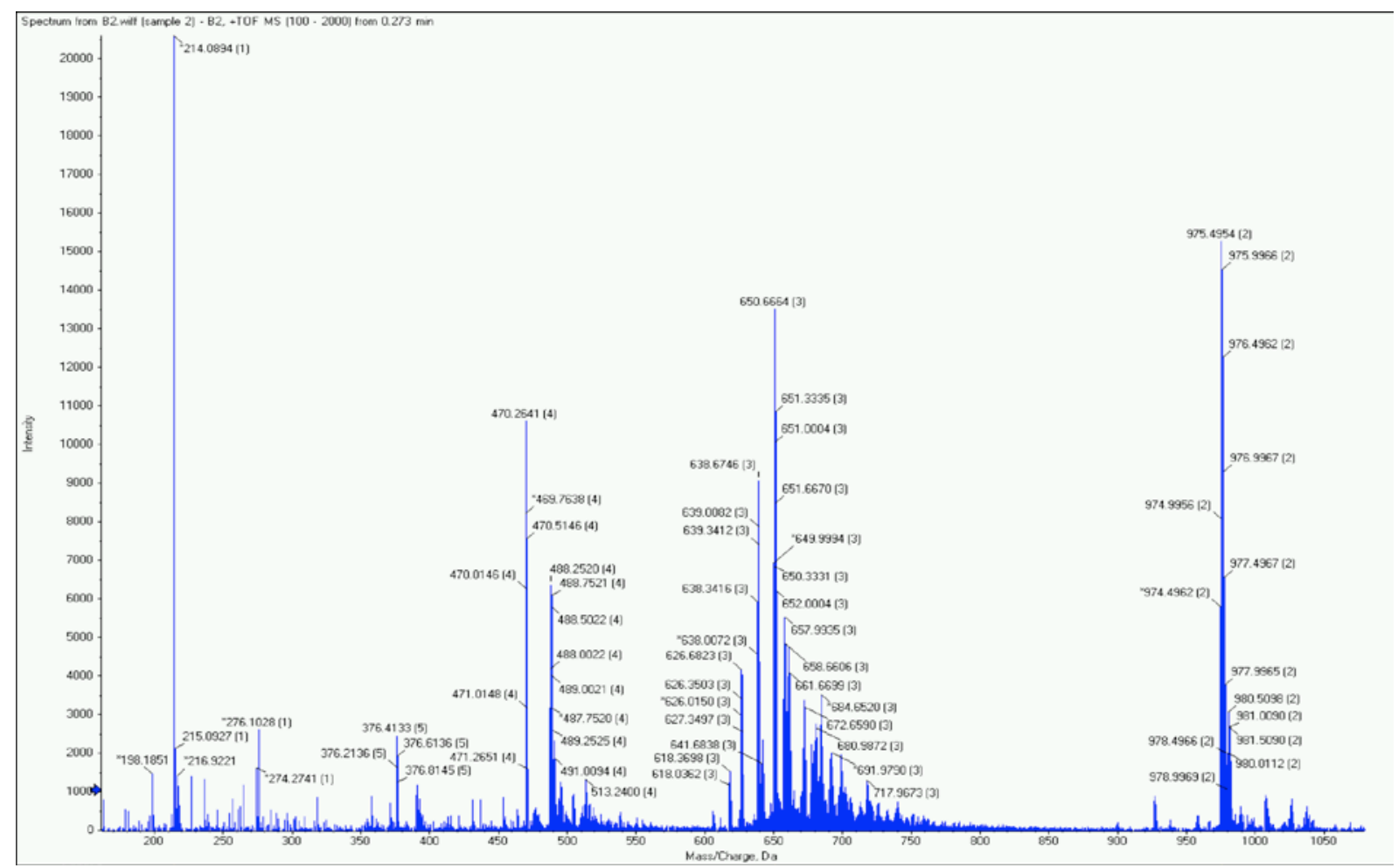

Figure S9: HR-ESI-MS spectrum of 7 in water/methanol, $1: 1(\mathrm{v} / \mathrm{v})$ containing 2 equiv of $\mathrm{Cu}\left(\mathrm{ClO}_{4}\right)_{2}$.

\begin{tabular}{ccc}
\hline & & $m / z \exp$. \\
\hline$[\mathrm{M}+2 \mathrm{Cu}]^{4+}$ & $\mathrm{C}_{104} \mathrm{H}_{152} \mathrm{~N}_{16} \mathrm{O}_{8}+2 \mathrm{Cu}^{2+}$ & 469.7638 \\
{$[\mathrm{M}+2 \mathrm{Cu}+\mathrm{Cl}]^{3+}$} & $\mathrm{C}_{104} \mathrm{H}_{152} \mathrm{~N}_{16} \mathrm{O}_{8}+2 \mathrm{Cu}^{2+}+\mathrm{Cl}^{-}$ & 638.0053 \\
{$[\mathrm{M}+2 \mathrm{Cu}+2 \mathrm{Cl}+\mathrm{H}]^{3+}$} & $\mathrm{C}_{104} \mathrm{H}_{152} \mathrm{~N}_{16} \mathrm{O}_{8}+2 \mathrm{Cu}^{2+}+2 \mathrm{Cl}^{-}+\mathrm{H}^{+}$ & 649.9988 \\
{$[\mathrm{M}+2 \mathrm{Cu}+2 \mathrm{Cl}]^{2+}$} & $\mathrm{C}_{104} \mathrm{H}_{152} \mathrm{~N}_{16} \mathrm{O}_{8}+2 \mathrm{Cu}^{2+}+2 \mathrm{Cl}^{-}$ & 974.4937 \\
\hline
\end{tabular}




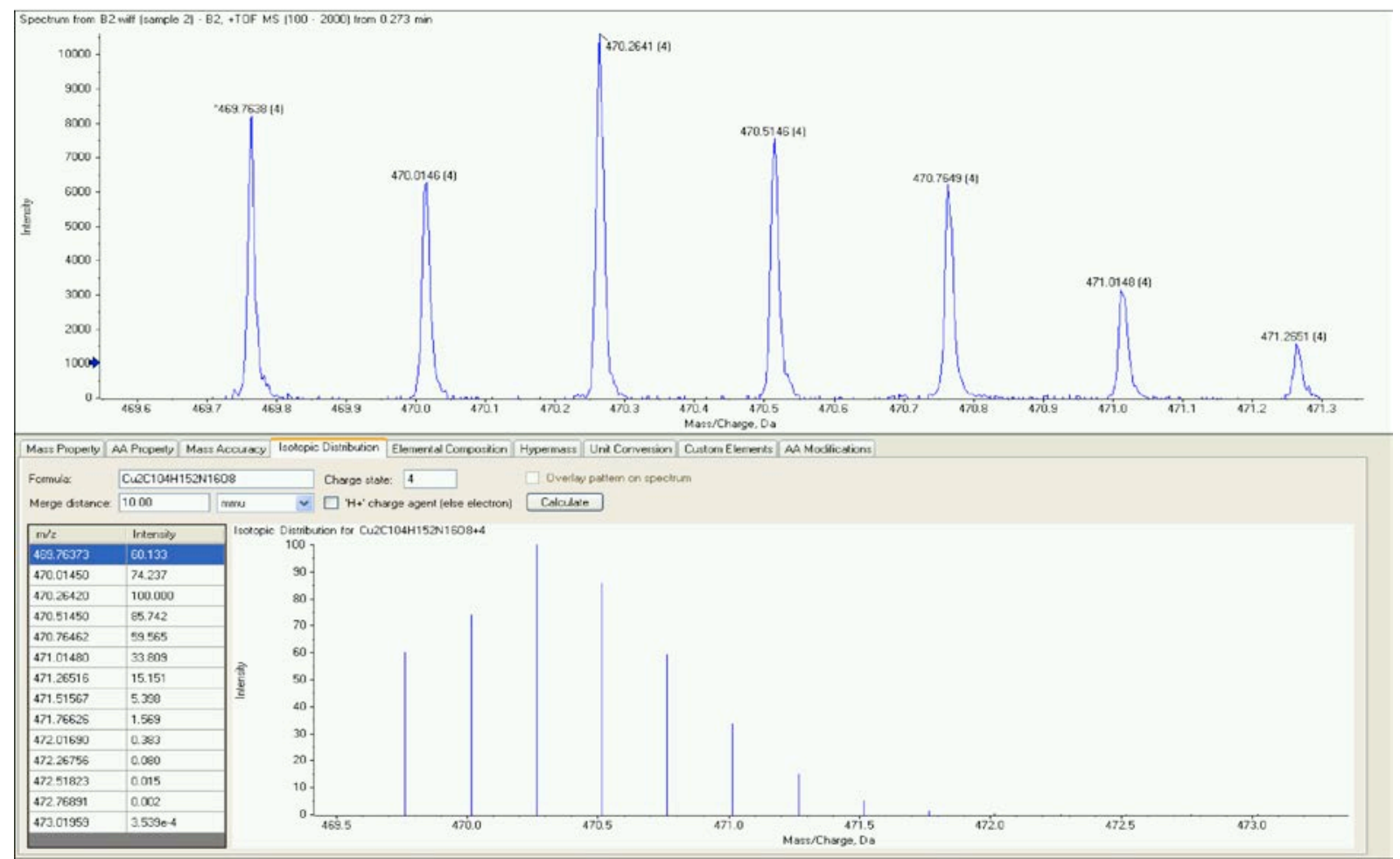

Figure S10: Experimental (top) and simulated (bottom) peaks for $\left[\mathrm{Cu}_{2} 7\right]^{4+}$.
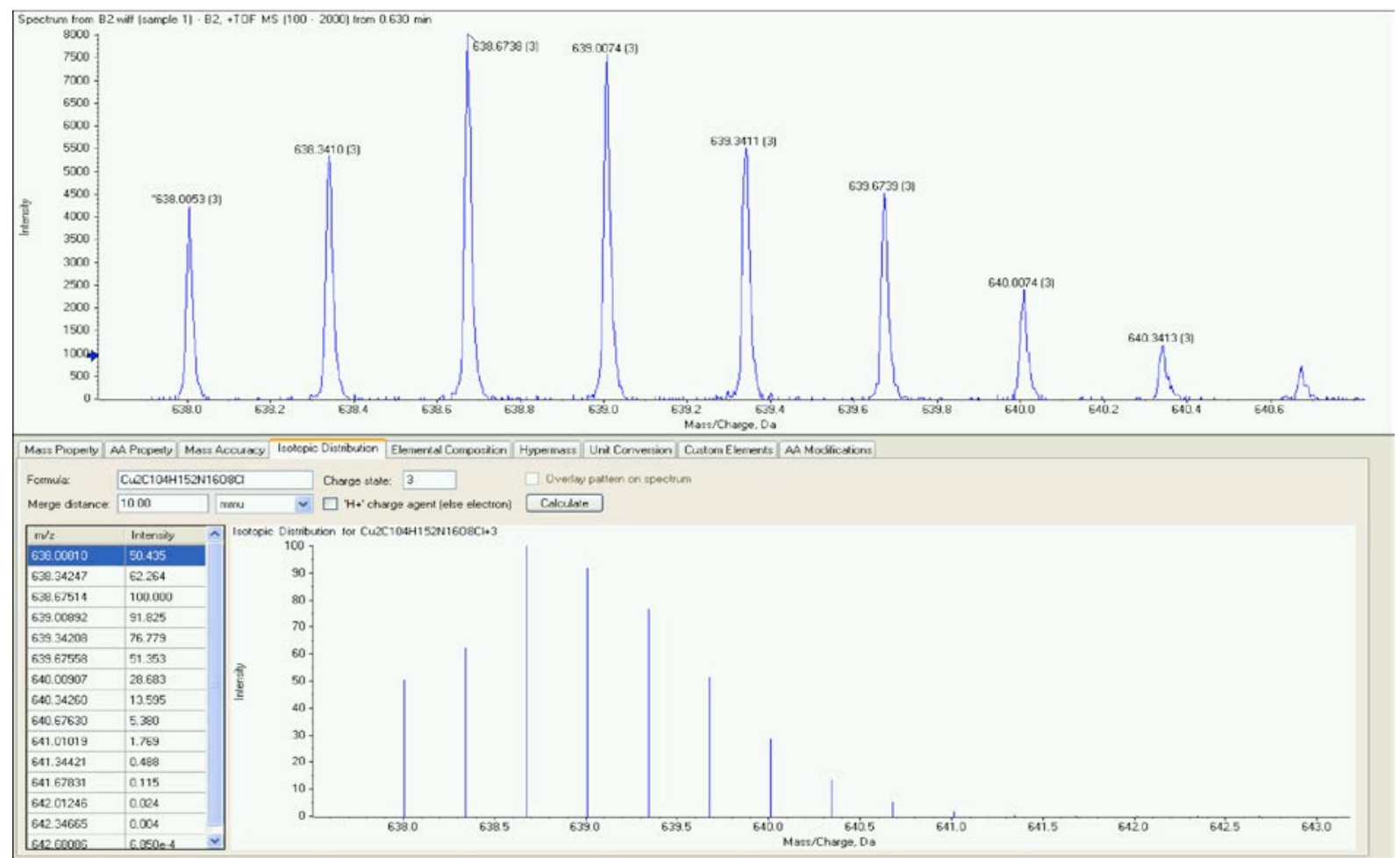

Figure S11: Experimental (top) and simulated (bottom) peaks for $\left[\mathrm{Cu}_{2} 7 \mathrm{Cl}\right]^{3+}$. 


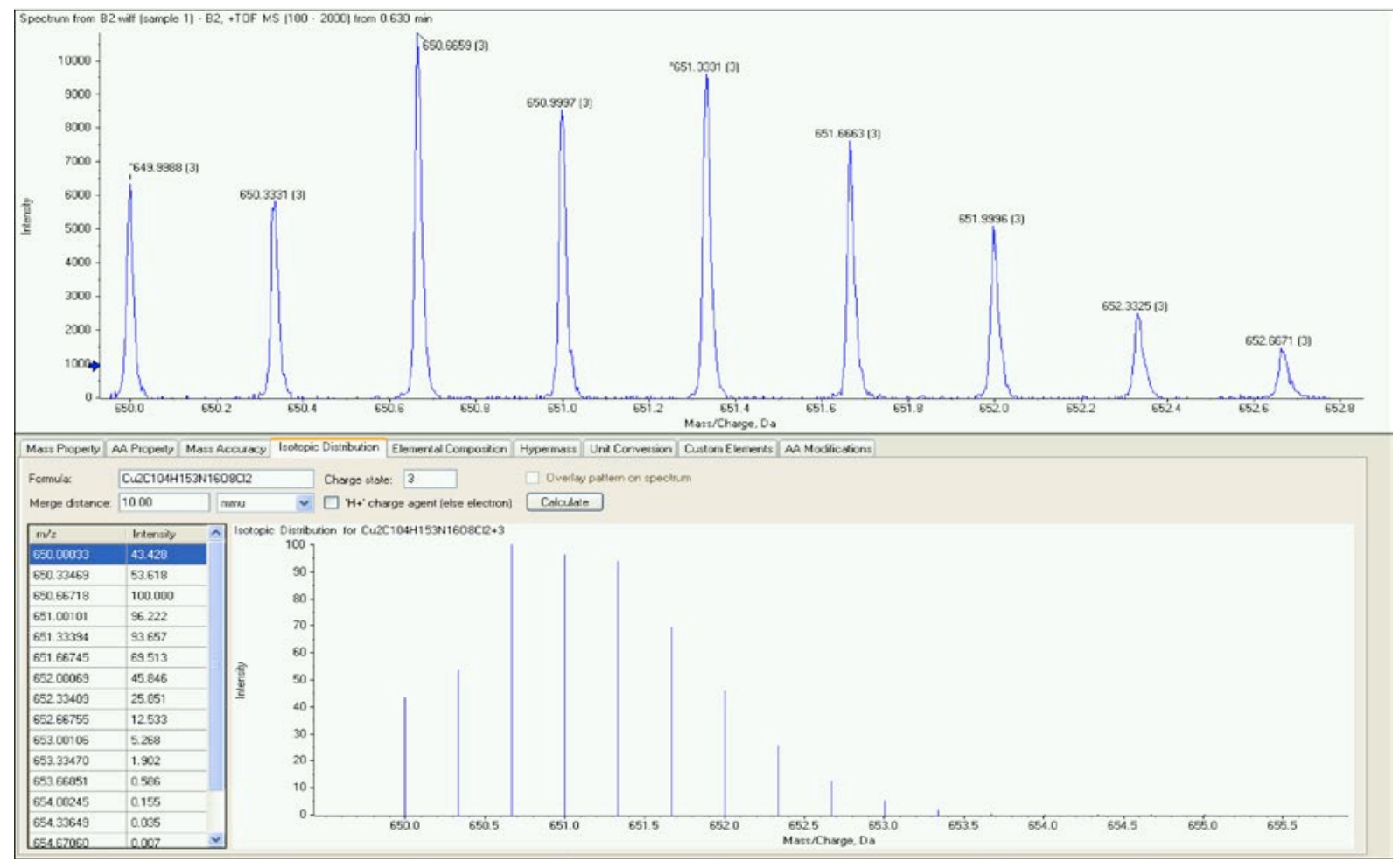

Figure S12: Experimental (top) and simulated (bottom) peaks for $\left[\mathrm{Cu}_{2}\left(\mathrm{H}_{7}\right) \mathrm{Cl}_{2}\right]^{3+}$.
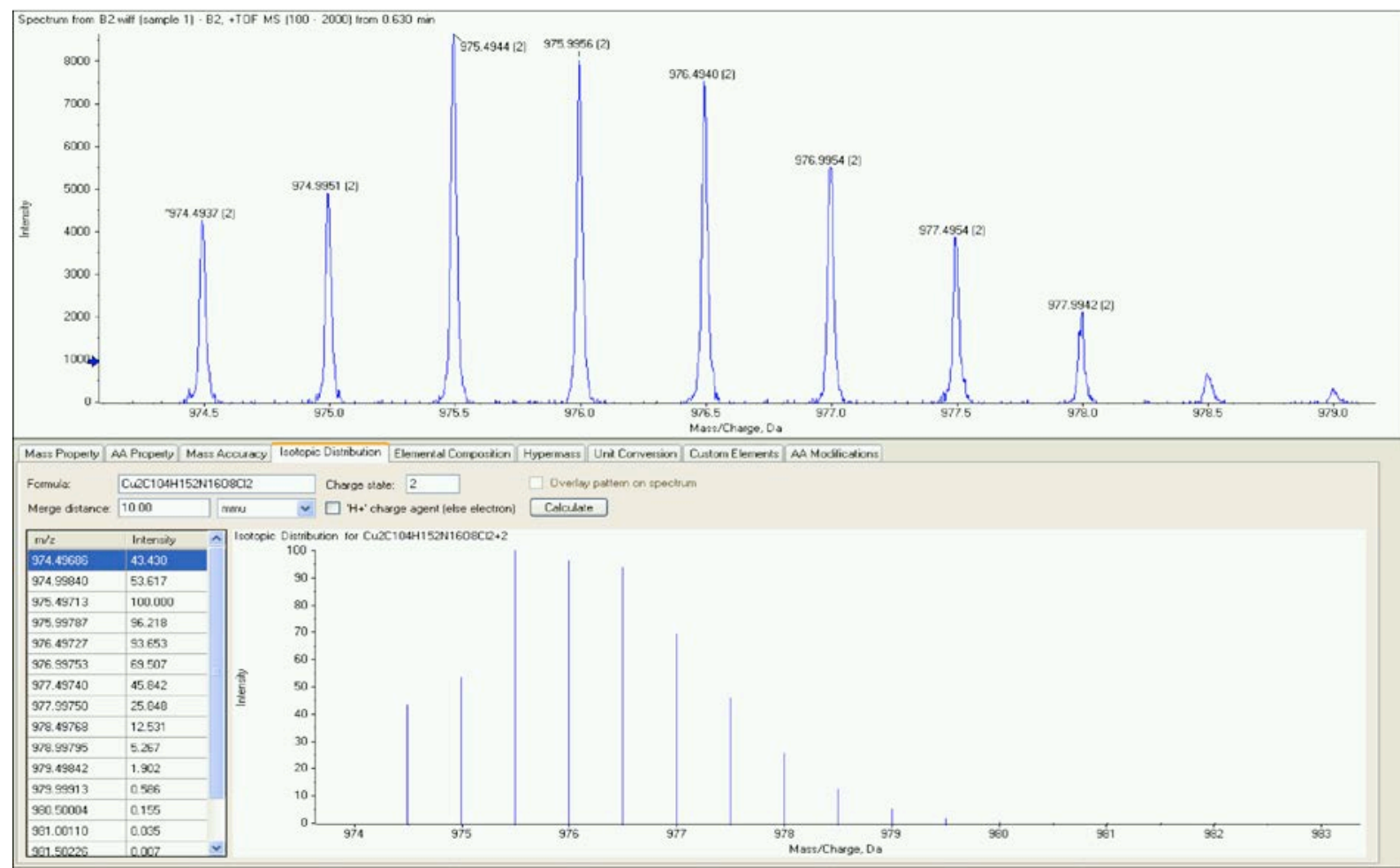

Figure S13: Experimental (top) and simulated (bottom) peaks for $\left[\mathrm{Cu}_{2} 7 \mathrm{Cl}_{2}\right]^{2+}$ 


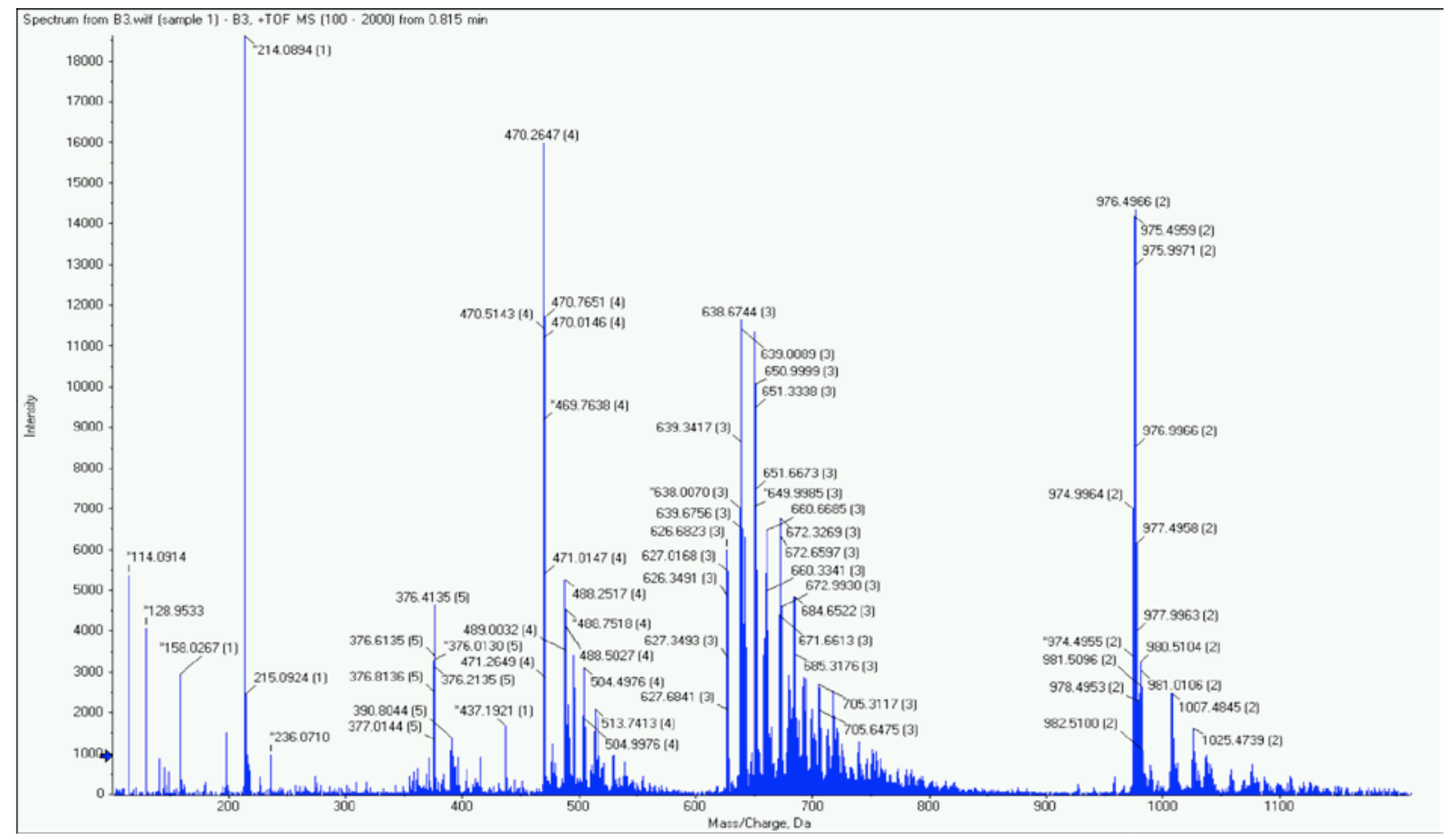

Figure S14: HR-ESI-MS spectrum of 7 in water/methanol, 1:1 (v/v) containing 3 equiv of $\mathrm{Cu}\left(\mathrm{ClO}_{4}\right)_{2}$. 


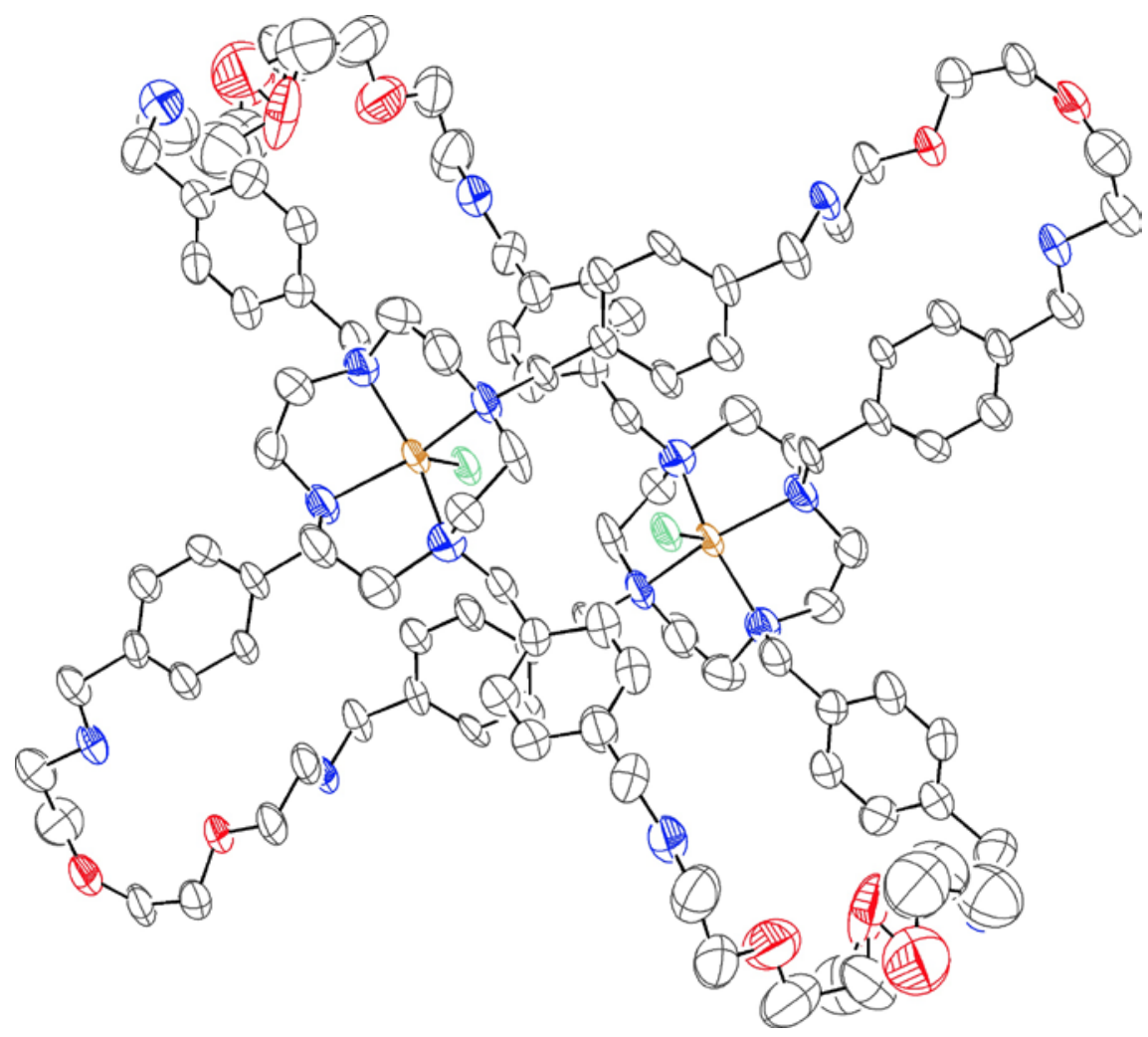

Figure S15: Ortep plot of $\left[\mathrm{Cu}_{2}\left(\mathrm{H}_{4} 7\right) \mathrm{Cl}_{2}\right] \mathrm{Cl}_{6} \cdot 9 \mathrm{H}_{2} \mathrm{O}$. Hydrogen atoms, water molecules not included into the cage, and chloride ions not coordinating to copper ions are omitted for clarity.

$\mathrm{C}_{104} \mathrm{H}_{170} \mathrm{Cl}_{8} \mathrm{Cu}_{2} \mathrm{~N}_{16} \mathrm{O}_{17}, M_{\mathrm{r}}=2327.23 \mathrm{~g} \cdot \mathrm{mol}^{-1}$, blue crystals, crystal size $0.27 \times 0.25 \times 0.15 \mathrm{~mm}^{3}$, monoclinic, space group $C 2 / \mathrm{c}, a=25.299(2) \AA, b=19.2324(13) \AA, c=28.254(4) \AA, \alpha=90^{\circ}, \beta=$ $91.806(9)^{\circ}, \gamma=90^{\circ}, V=13740.0(2) \AA^{3}, T=120 \mathrm{~K}, Z=4, D_{\text {calc }}=1.125 \mathrm{~g} / \mathrm{cm}^{3}, \lambda\left(\right.$ Mo-K $\left.\mathrm{K}_{\alpha}\right)=0.71073$ $\AA, \mu=523 \mathrm{~mm}^{-1}$, multi-scan absorption correction $\left(T_{\min }=0.53238, T_{\max }=1\right), 3.044^{\circ}<\theta<25^{\circ}$, 28875 measured reflections, 12024 independent reflections $\left(R_{\text {int }}=0.0978\right), 5280$ reflections with $I>$ $2 \sigma(I)$. Structure solved by direct methods (SHELXT) and refined by full-matrix least-squares (SHELXL) against $F^{2}$ to $R_{1}=0.1148 ;[I>2 \sigma(I)] w R_{2}=0.3454$ (all data), residual electron density $+1.380 /-1.243$ e $\AA^{-3}$.

Diffuse, disordered solvent molecules could not be adequately modeled. The bypass procedure SQUEEZE from PLATON software was used to remove the electronic contribution from these solvents. The total potential solvent accessible void volume was $2728 \AA^{3}$ and the electron count/cell $=572$. As the exact solvent content is not known, the reported formula reflects only the atoms used in the refinement. CCDC-1429442. 
Table S2: Selected bond distances and angles of complex $\left[\mathrm{Cu}_{2} \mathrm{H}_{4} 7 \mathrm{Cl}_{2}\right] \mathrm{Cl}_{6} \cdot 9 \mathrm{H}_{2} \mathrm{O}$.

\begin{tabular}{ccclllc}
\hline \multicolumn{3}{c}{ Bond Distances / } & \multicolumn{4}{l}{ Bond Angles / deg. } \\
\hline $\mathrm{Cu} 1$ & $\mathrm{C} 11$ & $2.3598(19)$ & $\mathrm{N} 2$ & $\mathrm{Cu} 1$ & $\mathrm{Cl1}$ & $104.57(17)$ \\
$\mathrm{Cu} 1$ & $\mathrm{~N} 2$ & $2.079(7)$ & $\mathrm{N} 2$ & $\mathrm{Cu} 1$ & $\mathrm{~N} 1$ & $87.0(3)$ \\
$\mathrm{Cu} 1$ & $\mathrm{~N} 1$ & $2.091(6)$ & $\mathrm{N} 1$ & $\mathrm{Cu} 1$ & $\mathrm{Cl1}$ & $107.41(17)$ \\
$\mathrm{Cu} 1$ & $\mathrm{~N} 3$ & $2.055(6)$ & $\mathrm{N} 3$ & $\mathrm{Cu} 1$ & $\mathrm{Cl1}$ & $104.38(17)$ \\
$\mathrm{Cu} 1$ & $\mathrm{~N} 4$ & $2.059(6)$ & $\mathrm{N} 3$ & $\mathrm{Cu} 1$ & $\mathrm{~N} 2$ & $85.4(2)$ \\
& & & $\mathrm{N} 3$ & $\mathrm{Cu} 1$ & $\mathrm{~N} 1$ & $148.2(2)$ \\
& & & $\mathrm{N} 3$ & $\mathrm{Cu} 1$ & $\mathrm{~N} 4$ & $86.5(3)$ \\
& & & $\mathrm{N} 4$ & $\mathrm{Cu} 1$ & $\mathrm{Cl1}$ & $104.76(17)$ \\
& & & $\mathrm{N} 4$ & $\mathrm{Cu} 1$ & $\mathrm{~N} 2$ & $150.7(2)$ \\
& & & $\mathrm{N} 4$ & $\mathrm{Cu} 1$ & $\mathrm{~N} 1$ & $85.2(3)$ \\
\hline
\end{tabular}




\section{$\underline{\text { References }}$}

1. This step was performed analogously to the following described procedures: Yoo, J.; Reichert, D. E.; Welch, M. J. J. Med. Chem. 2004, 47, 6625-6637. D'Aléo, A.; Xua, J.; Dob, K.; Muller, G.; Raymond, K. N. Helv. Chim. Acta 2009, 92, 2439-2460.

2. Iliashevsky, O.; Amir, L.; Glaser, R.; Marks, R. S.; Lemcoff, N. G. J. Mater. Chem. 2009, 19, $6616-6622$.

3. This reaction was performed analogously to a procedure described in: Liu, X.; Liu, Y.; Li, G.; Warmuth, R. Angew. Chem. Int. Ed. 2006, 45, 901-904.

4. García-España, E.; Ballester, M. J.; Lloret, F.; Moratal, J. M.; Faus, J.; Bianchi, A. J. Chem. Soc. Dalton Trans. 1988, 101-104.

5. Fontanelli M.; Micheloni, M. Proceedings of the 1st Spanish-Italian Congress on Thermodynamics of Metal Complexes, Diputación de Castellón, Castellón, Spain, 1990.

6. (a) Gran, G. Analyst 1952, 77, 661-671. (b) Rossotti, F. J.; Rossoti, H. J. Chem. Educ. 1965, $42,375-378$.

7. Gans, P.; Sabatini, A.; Vacca, A. Talanta 1996, 43, 1739-1753.

8. Hamilton, W. C. Statistics in Physical Chemistry, The Roland Press Co., New York, 1964.

9. Bologni, L.; Sabatini, A.; Vacca, A. Inorg. Chim. Acta 1983, 69, 71-75. 